Supporting Information. Table S1. Cation and Anion, Presence of Literature, and Experimental and Calculated Ionic Conductivities by Eq. (2).

\begin{tabular}{|c|c|c|c|c|c|c|c|c|c|c|c|c|c|c|}
\hline Cation & Anion & $\mathrm{IC} \mathrm{mS} / \mathrm{cm}$ & $\mathrm{T}{ }^{\circ} \mathrm{C}$ & $\mathrm{DP}$ & IP & LUMO & Area & Volume & Ovality & $\mathrm{N}$ & N2 & Eq.(2a) & $\mathrm{MR}(2 \mathrm{~b})$ & Lit. \\
\hline$\overline{B E I}$ & C4F9SO3 & 0.53 & 20 & 4.421 & 14.57 & -4.86 & 235.3 & 178.2 & 1.54 & 0.408 & 0.399 & 0.557 & 0.088 & (15) \\
\hline BEI & CF3COO & 2.5 & 20 & 4.421 & 14.57 & -4.86 & 235.3 & 178.2 & 1.54 & 0.408 & 0.399 & 3.896 & 2.929 & (15) \\
\hline BEI & CF3SO3 & 2.7 & 25 & 4.421 & 14.57 & -4.86 & 235.3 & 178.2 & 1.54 & 0.408 & 0.399 & 3.865 & 3.640 & (15) \\
\hline BMI & C3F7COO & 1 & 20 & 5.935 & 14.60 & -4.95 & 252.1 & 182.0 & 1.62 & 0.415 & 0.403 & 0.786 & -0.603 & (15) \\
\hline BMI & C4F9SO3 & 0.45 & 20 & 5.935 & 14.60 & -4.95 & 252.1 & 182.0 & 1.62 & 0.415 & 0.403 & 0.413 & 0.892 & (15) \\
\hline BMI & CF3COO & 3.2 & 20 & 5.935 & 14.60 & -4.95 & 252.1 & 182.0 & 1.62 & 0.415 & 0.403 & 3.297 & 3.733 & (15) \\
\hline BMI & PF6 & 1.46 & 25.5 & 5.935 & 14.60 & -4.95 & 252.1 & 182.0 & 1.62 & 0.415 & 0.403 & 1.586 & 1.441 & (15) \\
\hline BMI & CF3SO3 & 3.7 & 20 & 5.935 & 14.60 & -4.95 & 252.1 & 182.0 & 1.62 & 0.415 & 0.403 & 2.674 & 3.475 & (15) \\
\hline EMI & CF3SO3 & 9.2 & 25 & 1.757 & 14.95 & -4.98 & 217.6 & 143.7 & 1.64 & 0.416 & 0.394 & 8.919 & 9.350 & (15) \\
\hline EMI & BF4 & 13 & 22 & 1.757 & 14.95 & -4.98 & 217.6 & 143.7 & 1.64 & 0.416 & 0.394 & 12.821 & 10.937 & (15) \\
\hline EMI & CF3COO & 9.6 & 20 & 1.757 & 14.95 & -4.98 & 217.6 & 143.7 & 1.64 & 0.416 & 0.394 & 8.986 & 8.638 & (15) \\
\hline MPMI & TFSI & 2.52 & 25 & 3.309 & 14.52 & -4.87 & 209.0 & 167.4 & 1.42 & 0.400 & 0.393 & 4.495 & 2.396 & (15) \\
\hline EMI & C2F5BF3 & 1.295 & -30 & 1.757 & 14.95 & -4.98 & 217.6 & 143.7 & 1.64 & 0.416 & 0.394 & 1.342 & 0.651 & (16) \\
\hline EMI & C3F7BF3 & 1.456 & -20 & 1.757 & 14.95 & -4.98 & 217.6 & 143.7 & 1.64 & 0.416 & 0.394 & 1.369 & 0.217 & (16) \\
\hline EMI & TFSI & 3.365 & 0 & 1.757 & 14.95 & -4.98 & 217.6 & 143.7 & 1.64 & 0.416 & 0.394 & 3.433 & 4.826 & (16) \\
\hline EMI & C4F9BF3 & 1.822 & 0 & 1.757 & 14.95 & -4.98 & 217.6 & 143.7 & 1.64 & 0.416 & 0.394 & 1.881 & 1.657 & (16) \\
\hline EMI & C3F7BF3 & 2.334 & -10 & 1.757 & 14.95 & -4.98 & 217.6 & 143.7 & 1.64 & 0.416 & 0.394 & 2.241 & 2.156 & (16) \\
\hline EMI & CF3BF3 & 3.9 & -10 & 1.757 & 14.95 & -4.98 & 217.6 & 143.7 & 1.64 & 0.416 & 0.394 & 3.925 & 5.062 & (16) \\
\hline EMI & TFSI & 5.224 & 10 & 1.757 & 14.95 & -4.98 & 217.6 & 143.7 & 1.64 & 0.416 & 0.394 & 5.145 & 6.764 & (16) \\
\hline EMI & C3F7BF3 & 3.603 & 0 & 1.757 & 14.95 & -4.98 & 217.6 & 143.7 & 1.64 & 0.416 & 0.394 & 3.475 & 4.095 & (16) \\
\hline EMI & C4F9BF3 & 2.98 & 10 & 1.757 & 14.95 & -4.98 & 217.6 & 143.7 & 1.64 & 0.416 & 0.394 & 2.916 & 3.596 & (16) \\
\hline EMI & C3F7BF3 & 7.826 & 20 & 1.757 & 14.95 & -4.98 & 217.6 & 143.7 & 1.64 & 0.416 & 0.394 & 7.608 & 7.972 & (16) \\
\hline EMI & C4F9BF3 & 4.384 & 20 & 1.757 & 14.95 & -4.98 & 217.6 & 143.7 & 1.64 & 0.416 & 0.394 & 4.354 & 5.535 & (16) \\
\hline EMI & C4F9BF3 & 1.016 & -10 & 1.757 & 14.95 & -4.98 & 217.6 & 143.7 & 1.64 & 0.416 & 0.394 & 1.142 & -0.282 & (16) \\
\hline EMI & CF3BF3 & 12.49 & 20 & 1.757 & 14.95 & -4.98 & 217.6 & 143.7 & 1.64 & 0.416 & 0.394 & 12.595 & 10.878 & (16) \\
\hline EMI & C2F5BF3 & 2.382 & -20 & 1.757 & 14.95 & -4.98 & 217.6 & 143.7 & 1.64 & 0.416 & 0.394 & 2.234 & 2.590 & (16) \\
\hline EMI & C4F9BF3 & 0.49 & -20 & 1.757 & 14.95 & -4.98 & 217.6 & 143.7 & 1.64 & 0.416 & 0.394 & 0.621 & -2.220 & (16) \\
\hline EMI & TFSI & 8.876 & 25.24 & 1.757 & 14.95 & -4.98 & 217.6 & 143.7 & 1.64 & 0.416 & 0.394 & 9.108 & 9.719 & (16) \\
\hline EMI & C4F9BF3 & 5.402 & 25 & 1.757 & 14.95 & -4.98 & 217.6 & 143.7 & 1.64 & 0.416 & 0.394 & 5.266 & 6.504 & (16) \\
\hline EMI & TFSI & 1.927 & -10 & 1.757 & 14.95 & -4.98 & 217.6 & 143.7 & 1.64 & 0.416 & 0.394 & 2.212 & 2.887 & (16) \\
\hline EMI & BF4 & 9.399 & 15 & 1.757 & 14.95 & -4.98 & 217.6 & 143.7 & 1.64 & 0.416 & 0.394 & 9.940 & 9.580 & (16) \\
\hline EMI & C3F7BF3 & 5.595 & 10 & 1.757 & 14.95 & -4.98 & 217.6 & 143.7 & 1.64 & 0.416 & 0.394 & 5.206 & 6.033 & (16) \\
\hline EMI & C2F5BF3 & 3.784 & -10 & 1.757 & 14.95 & -4.98 & 217.6 & 143.7 & 1.64 & 0.416 & 0.394 & 3.509 & 4.529 & (16) \\
\hline EMI & CF3BF3 & 9.138 & 10.2 & 1.757 & 14.95 & -4.98 & 217.6 & 143.7 & 1.64 & 0.416 & 0.394 & 8.779 & 8.978 & (16) \\
\hline EMI & TFSI & 0.981 & -20 & 1.757 & 14.95 & -4.98 & 217.6 & 143.7 & 1.64 & 0.416 & 0.394 & 1.350 & 0.948 & (16) \\
\hline EMI & C2F5BF3 & 8.205 & 10 & 1.757 & 14.95 & -4.98 & 217.6 & 143.7 & 1.64 & 0.416 & 0.394 & 7.847 & 8.406 & (16) \\
\hline EMI & CF3BF3 & 6.253 & 0 & 1.757 & 14.95 & -4.98 & 217.6 & 143.7 & 1.64 & 0.416 & 0.394 & 5.919 & 7.001 & (16) \\
\hline EMI & CF3BF3 & 14.789 & 25 & 1.757 & 14.95 & -4.98 & 217.6 & 143.7 & 1.64 & 0.416 & 0.394 & 15.055 & 11.847 & (16) \\
\hline EMI & CF3BF3 & 1.975 & -20 & 1.757 & 14.95 & -4.98 & 217.6 & 143.7 & 1.64 & 0.416 & 0.394 & 2.517 & 3.123 & (16) \\
\hline EMI & C2F5BF3 & 5.783 & 0 & 1.757 & 14.95 & -4.98 & 217.6 & 143.7 & 1.64 & 0.416 & 0.394 & 5.315 & 6.468 & (16) \\
\hline EMI & C2F5BF3 & 10.803 & 20 & 1.757 & 14.95 & -4.98 & 217.6 & 143.7 & 1.64 & 0.416 & 0.394 & 11.363 & 10.345 & (16) \\
\hline HMI & $\mathrm{Cl}$ & 0.03 & 25 & 10.144 & 13.79 & -4.92 & 294.4 & 219.8 & 1.67 & 0.414 & 0.412 & 0.004 & 0.030 & (22) \\
\hline HMI & $\mathrm{Br}$ & 0.037 & 25 & 10.144 & 13.79 & -4.92 & 294.4 & 219.8 & 1.67 & 0.414 & 0.412 & 0.004 & 0.037 & (22) \\
\hline BMI & C2F5BF3 & 5.5 & 25 & 5.935 & 14.60 & -4.95 & 252.1 & 182.0 & 1.62 & 0.415 & 0.403 & 5.139 & 6.409 & (22) \\
\hline BMI & CF3BF3 & 5.9 & 25 & 5.935 & 14.60 & -4.95 & 252.1 & 182.0 & 1.62 & 0.415 & 0.403 & 5.724 & 6.942 & (22) \\
\hline
\end{tabular}




\begin{tabular}{|c|c|c|c|c|c|c|c|c|c|c|c|c|c|c|}
\hline Cation & Anion & $\mathrm{IC} \mathrm{mS} / \mathrm{cm}$ & $\mathrm{T}^{\circ} \mathrm{C}$ & $\mathrm{DP}$ & IP & LUMO & Area & Volume & Ovality & $\mathrm{N}$ & N2 & Eq.(2a) & $\mathrm{MR}(2 \mathrm{~b})$ & Lit. \\
\hline EEE-EOM & CF3BF3 & 2 & 25 & 4.120 & 14.14 & -4.23 & 195.2 & 181.7 & 1.26 & 0.553 & 0.000 & 1.778 & 2.883 & $\overline{(22)}$ \\
\hline EEE-EOM & C2F5BF3 & 2.4 & 25 & 4.120 & 14.14 & -4.23 & 195.2 & 181.7 & 1.26 & 0.553 & 0.000 & 1.564 & 2.350 & (22) \\
\hline EEE-EOM & TFSI & 2.1 & 25 & 4.120 & 14.14 & -4.23 & 195.2 & 181.7 & 1.26 & 0.553 & 0.000 & 0.897 & 0.708 & (22) \\
\hline EMI & $\mathrm{CH} 3 \mathrm{COO}$ & 2.8 & 20 & 1.757 & 14.95 & -4.98 & 217.6 & 143.7 & 1.64 & 0.416 & 0.394 & 2.797 & 2.800 & (22) \\
\hline EMI & C4F9BF3 & 5.2 & 25 & 1.757 & 14.95 & -4.98 & 217.6 & 143.7 & 1.64 & 0.416 & 0.394 & 5.266 & 6.504 & (22) \\
\hline EMI & C3F7COO & 2.7 & 20 & 1.757 & 14.95 & -4.98 & 217.6 & 143.7 & 1.64 & 0.416 & 0.394 & 2.708 & 4.303 & (22) \\
\hline EMI & CF3SO2NCOCF3 & 9.8 & 25 & 1.757 & 14.95 & -4.98 & 217.6 & 143.7 & 1.64 & 0.416 & 0.394 & 9.765 & 9.800 & (22) \\
\hline EMI & BF4 & 13.6 & 25 & 1.757 & 14.95 & -4.98 & 217.6 & 143.7 & 1.64 & 0.416 & 0.394 & 14.266 & 11.519 & (22) \\
\hline EMI & CF3BF3 & 14.8 & 25 & 1.757 & 14.95 & -4.98 & 217.6 & 143.7 & 1.64 & 0.416 & 0.394 & 15.055 & 11.847 & (22) \\
\hline EMI & C3F7BF3 & 8.6 & 25 & 1.757 & 14.95 & -4.98 & 217.6 & 143.7 & 1.64 & 0.416 & 0.394 & 9.131 & 8.941 & (22) \\
\hline EMI & C2F5BF3 & 12 & 25 & 1.757 & 14.95 & -4.98 & 217.6 & 143.7 & 1.64 & 0.416 & 0.394 & 13.592 & 11.314 & (22) \\
\hline MEE3 & TFSI & 2.2 & 25 & 1.855 & 15.63 & -4.26 & 174.9 & 162.2 & 1.22 & 0.565 & 0.000 & 2.059 & 1.705 & (22) \\
\hline MEE4 & TFSI & 1.6 & 25 & 3.766 & 14.87 & -4.22 & 191.4 & 172.1 & 1.28 & 0.565 & 0.000 & 1.399 & 2.096 & (22) \\
\hline MEE4 & C2F5BF3 & 2.3 & 25 & 3.766 & 14.87 & -4.22 & 191.4 & 172.1 & 1.28 & 0.565 & 0.000 & 2.306 & 3.738 & (22) \\
\hline MEE4 & CF3BF3 & 2.1 & 25 & 3.766 & 14.87 & -4.22 & 191.4 & 172.1 & 1.28 & 0.565 & 0.000 & 2.598 & 4.271 & (22) \\
\hline MEE-EOM & CF3BF3 & 3 & 25 & 4.531 & 14.23 & -4.31 & 182.5 & 165.5 & 1.25 & 0.569 & 0.000 & 2.413 & 3.016 & (22) \\
\hline MEE-EOM & C2F5BF3 & 3.2 & 25 & 4.531 & 14.23 & -4.31 & 182.5 & 165.5 & 1.25 & 0.569 & 0.000 & 2.139 & 2.483 & (22) \\
\hline MEE-EOM & TFSI & 2.6 & 25 & 4.531 & 14.23 & -4.31 & 182.5 & 165.5 & 1.25 & 0.569 & 0.000 & 1.286 & 0.841 & (22) \\
\hline MEE-EOM & BF4 & 1.3 & 25 & 4.531 & 14.23 & -4.31 & 182.5 & 165.5 & 1.25 & 0.569 & 0.000 & 2.265 & 2.688 & (22) \\
\hline MI-EOM & C2F5BF3 & 6.1 & 25 & 6.392 & 13.91 & -5.01 & 246.2 & 171.3 & 1.65 & 0.415 & 0.408 & 5.227 & 7.108 & (22) \\
\hline MI-EOM & CF3BF3 & 6.9 & 25 & 6.392 & 13.91 & -5.01 & 246.2 & 171.3 & 1.65 & 0.415 & 0.408 & 5.821 & 7.641 & (22) \\
\hline MME-EOM & C2F5BF3 & 3.8 & 25 & 4.613 & 14.29 & -4.40 & 169.4 & 146.8 & 1.26 & 0.593 & 0.000 & 3.258 & 3.259 & (22) \\
\hline MME-EOM & TFSI & 3.1 & 25 & 4.613 & 14.29 & -4.40 & 169.4 & 146.8 & 1.26 & 0.593 & 0.000 & 2.042 & 1.617 & (22) \\
\hline MME-EOM & CF3BF3 & 2.5 & 25 & 4.613 & 14.29 & -4.40 & 169.4 & 146.8 & 1.26 & 0.593 & 0.000 & 3.648 & 3.792 & (22) \\
\hline MME-EOM & $\mathrm{BF} 4$ & 1.7 & 25 & 4.613 & 14.29 & -4.40 & 169.4 & 146.8 & 1.26 & 0.593 & 0.000 & 3.438 & 3.463 & (22) \\
\hline MMM3 & TFSI & 3.3 & 25 & 3.449 & 15.76 & -4.45 & 147.6 & 127.8 & 1.20 & 0.606 & 0.000 & 2.691 & 2.054 & (22) \\
\hline MMM4 & TFSI & 2.1 & 25 & 5.689 & 14.96 & -4.43 & 164.2 & 142.1 & 1.25 & 0.606 & 0.000 & 1.739 & 1.947 & (22) \\
\hline PMI & C2F5BF3 & 7.5 & 25 & 3.777 & 14.92 & -4.97 & 239.8 & 177.4 & 1.57 & 0.415 & 0.403 & 7.343 & 6.430 & (22) \\
\hline PMI & CF3BF3 & 8.5 & 25 & 3.777 & 14.92 & -4.97 & 239.8 & 177.4 & 1.57 & 0.415 & 0.403 & 8.157 & 6.963 & (22) \\
\hline PPR12(c-C5N) & TFSI & 1.2 & 25 & 2.474 & 15.74 & -4.22 & 160.5 & 151.1 & 1.17 & 0.566 & 0.000 & 1.379 & 0.929 & (22) \\
\hline PPR13(c-C5N) & TFSI & 1.5 & 25 & 2.649 & 15.56 & -4.18 & 176.7 & 165.9 & 1.21 & 0.573 & 0.000 & 1.293 & 1.866 & (22) \\
\hline PPR14(c-C5N) & TFSI & 1.1 & 25 & 3.700 & 14.86 & -4.17 & 195.8 & 183.5 & 1.25 & 0.576 & 0.000 & 0.964 & 1.993 & (22) \\
\hline PRL13(c-C4N) & TFSI & 3.6 & 25 & 1.524 & 15.67 & -4.23 & 167.0 & 151.0 & 1.22 & 0.569 & 0.000 & 3.002 & 2.160 & (22) \\
\hline PRL14(c-C4N) & C2F5BF3 & 3.5 & 25 & 3.455 & 14.89 & -4.21 & 185.8 & 168.3 & 1.26 & 0.569 & 0.000 & 2.452 & 3.632 & (22) \\
\hline PRL1-EOM(c-C4N) & C2F5BF3 & 4.5 & 25 & 3.489 & 14.24 & -4.28 & 177.6 & 156.8 & 1.26 & 0.576 & 0.000 & 3.316 & 3.338 & (22) \\
\hline PZB & BF4 & 2.5 & 25 & 4.742 & 14.77 & -5.80 & 278.2 & 190.0 & 1.74 & 0.493 & 0.000 & 3.188 & 2.657 & (22) \\
\hline BBB6 & TFSI & 0.16 & 25 & 3.736 & 13.76 & -4.02 & 349.7 & 328.6 & 1.52 & 0.564 & 0.000 & 0.510 & 1.451 & (24) \\
\hline BBB7 & TFSI & 0.16 & 25 & 5.465 & 13.41 & -4.02 & 368.3 & 345.7 & 1.55 & 0.564 & 0.000 & 0.267 & 0.485 & (24) \\
\hline BBB8 & TFSI & 0.13 & 25 & 7.376 & 13.14 & -4.02 & 386.9 & 362.8 & 1.57 & 0.564 & 0.000 & 0.113 & -0.526 & (24) \\
\hline BBB8 & CF3SO3 & 0.017 & 25 & 7.376 & 13.14 & -4.02 & 386.9 & 362.8 & 1.57 & 0.564 & 0.000 & 0.106 & -0.848 & (24) \\
\hline BMI & PF6 & 2.47 & 35 & 5.935 & 14.60 & -4.95 & 252.1 & 182.0 & 1.62 & 0.415 & 0.403 & 2.373 & 3.283 & (24) \\
\hline BMI & TFSI & 9.24 & 50 & 5.935 & 14.60 & -4.95 & 252.1 & 182.0 & 1.62 & 0.415 & 0.403 & 8.223 & 9.614 & (24) \\
\hline BMI & TFSI & 2.65 & 15 & 5.935 & 14.60 & -4.95 & 252.1 & 182.0 & 1.62 & 0.415 & 0.403 & 2.198 & 2.828 & (24) \\
\hline BMI & TFSI & 3.31 & 20 & 5.935 & 14.60 & -4.95 & 252.1 & 182.0 & 1.62 & 0.415 & 0.403 & 2.711 & 3.798 & (24) \\
\hline BMI & TFSI & 4.06 & 25 & 5.935 & 14.60 & -4.95 & 252.1 & 182.0 & 1.62 & 0.415 & 0.403 & 3.314 & 4.767 & (24) \\
\hline BMI & PF6 & 1.46 & 25 & 5.935 & 14.60 & -4.95 & 252.1 & 182.0 & 1.62 & 0.415 & 0.403 & 1.551 & 1.344 & (24) \\
\hline
\end{tabular}




\begin{tabular}{|c|c|c|c|c|c|c|c|c|c|c|c|c|c|c|}
\hline Cation & Anion & IC $\mathrm{mS} / \mathrm{cm}$ & $\mathrm{T}^{\circ} \mathrm{C}$ & DP & IP & LUMO & Area & Volume & Ovality & $\mathrm{N}$ & N2 & Eq.(2a) & $\mathrm{MR}(2 \mathrm{~b})$ & Lit. \\
\hline BMI & PF6 & 1.09 & 20 & 5.935 & 14.60 & -4.95 & 252.1 & 182.0 & 1.62 & 0.415 & 0.403 & 1.227 & 0.375 & (24) \\
\hline BMI & PF6 & 0.79 & 15 & 5.935 & 14.60 & -4.95 & 252.1 & 182.0 & 1.62 & 0.415 & 0.403 & 0.951 & -0.594 & (24) \\
\hline BMI & TFSI & 5.85 & 35 & 5.935 & 14.60 & -4.95 & 252.1 & 182.0 & 1.62 & 0.415 & 0.403 & 4.844 & 6.706 & (24) \\
\hline BMI & PF6 & 4.77 & 50 & 5.935 & 14.60 & -4.95 & 252.1 & 182.0 & 1.62 & 0.415 & 0.403 & 4.188 & 6.191 & (24) \\
\hline BMI & BF4 & 3.5 & 25 & 5.935 & 14.60 & -4.95 & 252.1 & 182.0 & 1.62 & 0.415 & 0.403 & 5.409 & 6.613 & (24) \\
\hline EEE6 & TFSI & 0.67 & 25 & 8.264 & 13.79 & -4.16 & 239.5 & 225.2 & 1.34 & 0.543 & 0.000 & 0.124 & 0.525 & (24) \\
\hline EEE7 & TFSI & 0.51 & 25 & 9.910 & 13.44 & -4.14 & 263.7 & 248.9 & 1.38 & 0.545 & 0.000 & 0.000 & 0.260 & (24) \\
\hline EEE8 & TFSI & 0.33 & 25 & 12.926 & 13.16 & -4.16 & 278.2 & 260.3 & 1.41 & 0.547 & 0.000 & -0.044 & -0.468 & (24) \\
\hline EiPiP7 & TFSI & 0.31 & 25 & 8.984 & 13.40 & -4.04 & 279.2 & 274.0 & 1.37 & 0.531 & 0.000 & -0.360 & 0.496 & (24) \\
\hline EMI & EtOSO3 & 4.85 & 30 & 1.757 & 14.95 & -4.98 & 217.6 & 143.7 & 1.64 & 0.416 & 0.394 & 4.798 & 6.299 & (24) \\
\hline EMI & TFSI & 9.12 & 25 & 1.757 & 14.95 & -4.98 & 217.6 & 143.7 & 1.64 & 0.416 & 0.394 & 9.029 & 9.673 & (24) \\
\hline EMI & EtOSO3 & 13.51 & 60 & 1.757 & 14.95 & -4.98 & 217.6 & 143.7 & 1.64 & 0.416 & 0.394 & 13.532 & 12.115 & (24) \\
\hline EMI & BF4 & 2.45 & -15 & 1.757 & 14.95 & -4.98 & 217.6 & 143.7 & 1.64 & 0.416 & 0.394 & 2.974 & 3.764 & (24) \\
\hline EMI & TFSI & 7.73 & 20 & 1.757 & 14.95 & -4.98 & 217.6 & 143.7 & 1.64 & 0.416 & 0.394 & 7.522 & 8.703 & (24) \\
\hline EMI & TFSI & 6.44 & 15 & 1.757 & 14.95 & -4.98 & 217.6 & 143.7 & 1.64 & 0.416 & 0.394 & 6.238 & 7.734 & (24) \\
\hline EMI & EtOSO3 & 3.82 & 25 & 1.757 & 14.95 & -4.98 & 217.6 & 143.7 & 1.64 & 0.416 & 0.394 & 3.970 & 5.329 & (24) \\
\hline EMI & EtOSO3 & 6.06 & 35 & 1.757 & 14.95 & -4.98 & 217.6 & 143.7 & 1.64 & 0.416 & 0.394 & 5.764 & 7.268 & (24) \\
\hline EMI & EtOSO3 & 15.38 & 65 & 1.757 & 14.95 & -4.98 & 217.6 & 143.7 & 1.64 & 0.416 & 0.394 & 15.890 & 13.084 & (24) \\
\hline EMI & EtOSO3 & 7.24 & 40 & 1.757 & 14.95 & -4.98 & 217.6 & 143.7 & 1.64 & 0.416 & 0.394 & 6.891 & 8.238 & (24) \\
\hline EMI & BF4 & 2.96 & -10 & 1.757 & 14.95 & -4.98 & 217.6 & 143.7 & 1.64 & 0.416 & 0.394 & 3.701 & 4.733 & (24) \\
\hline EMI & EtOSO3 & 11.78 & 55 & 1.757 & 14.95 & -4.98 & 217.6 & 143.7 & 1.64 & 0.416 & 0.394 & 11.490 & 11.146 & (24) \\
\hline EMI & BF4 & 4.45 & -5 & 1.757 & 14.95 & -4.98 & 217.6 & 143.7 & 1.64 & 0.416 & 0.394 & 4.566 & 5.702 & (24) \\
\hline EMI & EtOSO3 & 10.1 & 50 & 1.757 & 14.95 & -4.98 & 217.6 & 143.7 & 1.64 & 0.416 & 0.394 & 9.725 & 10.176 & (24) \\
\hline EMI & EtOSO3 & 8.61 & 45 & 1.757 & 14.95 & -4.98 & 217.6 & 143.7 & 1.64 & 0.416 & 0.394 & 8.202 & 9.207 & (24) \\
\hline EMI & EtOSO3 & 0.286 & -15 & 1.757 & 14.95 & -4.98 & 217.6 & 143.7 & 1.64 & 0.416 & 0.394 & 0.554 & -2.425 & (24) \\
\hline EMI & EtOSO3 & 2.91 & 20 & 1.757 & 14.95 & -4.98 & 217.6 & 143.7 & 1.64 & 0.416 & 0.394 & 3.264 & 4.360 & (24) \\
\hline EMI & EtOSO3 & 0.374 & -10 & 1.757 & 14.95 & -4.98 & 217.6 & 143.7 & 1.64 & 0.416 & 0.394 & 0.774 & -1.456 & (24) \\
\hline EMI & TFSI & 12.29 & 35 & 1.757 & 14.95 & -4.98 & 217.6 & 143.7 & 1.64 & 0.416 & 0.394 & 12.855 & 11.611 & (24) \\
\hline EMI & EtOSO3 & 0.495 & -5 & 1.757 & 14.95 & -4.98 & 217.6 & 143.7 & 1.64 & 0.416 & 0.394 & 1.036 & -0.487 & (24) \\
\hline EMI & EtOSO3 & 2.02 & 15 & 1.757 & 14.95 & -4.98 & 217.6 & 143.7 & 1.64 & 0.416 & 0.394 & 2.661 & 3.391 & (24) \\
\hline EMI & EtOSO3 & 0.727 & 0 & 1.757 & 14.95 & -4.98 & 217.6 & 143.7 & 1.64 & 0.416 & 0.394 & 1.347 & 0.483 & (24) \\
\hline EMI & EtOSO3 & 1.051 & 5 & 1.757 & 14.95 & -4.98 & 217.6 & 143.7 & 1.64 & 0.416 & 0.394 & 1.714 & 1.452 & (24) \\
\hline EMI & EtOSO3 & 1.388 & 10 & 1.757 & 14.95 & -4.98 & 217.6 & 143.7 & 1.64 & 0.416 & 0.394 & 2.149 & 2.421 & (24) \\
\hline EMI & BF4 & 6.12 & 0 & 1.757 & 14.95 & -4.98 & 217.6 & 143.7 & 1.64 & 0.416 & 0.394 & 5.593 & 6.672 & (24) \\
\hline EMI & BF4 & 7.35 & 5 & 1.757 & 14.95 & -4.98 & 217.6 & 143.7 & 1.64 & 0.416 & 0.394 & 6.810 & 7.641 & (24) \\
\hline EMI & BF4 & 8.96 & 10 & 1.757 & 14.95 & -4.98 & 217.6 & 143.7 & 1.64 & 0.416 & 0.394 & 8.246 & 8.611 & (24) \\
\hline EMI & BF4 & 10.91 & 15 & 1.757 & 14.95 & -4.98 & 217.6 & 143.7 & 1.64 & 0.416 & 0.394 & 9.940 & 9.580 & (24) \\
\hline EMI & BF4 & 13.05 & 20 & 1.757 & 14.95 & -4.98 & 217.6 & 143.7 & 1.64 & 0.416 & 0.394 & 11.931 & 10.549 & (24) \\
\hline EMI & BF4 & 15.71 & 25 & 1.757 & 14.95 & -4.98 & 217.6 & 143.7 & 1.64 & 0.416 & 0.394 & 14.266 & 11.519 & (24) \\
\hline HMI & TFSI & 1.35 & 15 & 10.144 & 13.79 & -4.92 & 294.4 & 219.8 & 1.67 & 0.414 & 0.412 & 1.276 & -0.617 & (24) \\
\hline HMI & TFSI & 1.73 & 20 & 10.144 & 13.79 & -4.92 & 294.4 & 219.8 & 1.67 & 0.414 & 0.412 & 1.614 & 0.352 & (24) \\
\hline HMI & TFSI & 2.18 & 25 & 10.144 & 13.79 & -4.92 & 294.4 & 219.8 & 1.67 & 0.414 & 0.412 & 2.010 & 1.322 & (24) \\
\hline HMI & TFSI & 3.27 & 35 & 10.144 & 13.79 & -4.92 & 294.4 & 219.8 & 1.67 & 0.414 & 0.412 & 3.016 & 3.261 & (24) \\
\hline HMI & TFSI & 5.46 & 50 & 10.144 & 13.79 & -4.92 & 294.4 & 219.8 & 1.67 & 0.414 & 0.412 & 5.238 & 6.169 & (24) \\
\hline MMM6 & TFSI & 0.43 & 25 & 10.692 & 13.85 & -4.42 & 201.4 & 176.4 & 1.32 & 0.607 & 0.000 & 0.820 & 1.170 & (24) \\
\hline MMM7 & TFSI & 0.4 & 25 & 13.348 & 13.49 & -4.42 & 223.2 & 197.2 & 1.36 & 0.607 & 0.000 & 0.555 & 0.606 & (24) \\
\hline
\end{tabular}




\begin{tabular}{|c|c|c|c|c|c|c|c|c|c|c|c|c|c|c|}
\hline Cation & Anion & IC $\mathrm{mS} / \mathrm{cm}$ & $\mathrm{T}^{\circ} \mathrm{C}$ & DP & IP & LUMO & Area & Volume & Ovality & $\mathrm{N}$ & N2 & Eq.(2a) & $\mathrm{MR}(2 \mathrm{~b})$ & Lit. \\
\hline MMM8 & TFSI & 0.35 & 25 & 16.047 & 13.20 & -4.42 & 238.6 & 210.6 & 1.39 & 0.607 & 0.000 & 0.408 & 0.044 & (24) \\
\hline PMI & $\mathrm{BF} 4$ & 5.9 & 25 & 3.777 & 14.92 & -4.97 & 239.8 & 177.4 & 1.57 & 0.415 & 0.403 & 7.718 & 6.634 & (24) \\
\hline \multicolumn{15}{|l|}{ Prediction Data Set } \\
\hline M888 & TFSI & 0.048 & 25 & 10.441 & 13.14 & -4.13 & 486.6 & 451.3 & 1.71 & 0.580 & 0.000 & 0.056 & -5.067 & (22) \\
\hline $8 \mathrm{MI}$ & BF4 & 0.576 & 25 & 14.952 & 13.20 & -4.92 & 310.6 & 262.0 & 1.57 & 0.414 & 0.413 & 1.924 & -0.745 & (22) \\
\hline $\mathrm{PZB}(\mathrm{p}-\mathrm{Me})$ & BF4 & 1.81 & 25 & 3.653 & 14.71 & -5.67 & 272.2 & 212.6 & 1.58 & 0.470 & 0.000 & 3.083 & -1.069 & (22) \\
\hline $\mathrm{PZB}(\mathrm{m}-\mathrm{Me})$ & EtOSO3 & 2.18 & 25 & 3.769 & 14.73 & -5.66 & 319.0 & 221.1 & 1.80 & 0.511 & 0.000 & 0.909 & -4.918 & (22) \\
\hline $\mathrm{PZB}(\mathrm{m}-\mathrm{Me})$ & C4F9SO3 & 1.302 & 25 & 3.769 & 14.73 & -5.66 & 319.0 & 221.1 & 1.80 & 0.511 & 0.000 & 0.353 & -3.481 & (22) \\
\hline EEE5 & TFSI & 1.82 & 25 & 5.364 & 14.26 & -4.15 & 225.9 & 214.2 & 1.30 & 0.545 & 0.000 & 0.288 & 1.193 & (20) \\
\hline MEE5 & TFSI & 2.17 & 25 & 5.581 & 14.29 & -4.24 & 213.5 & 196.1 & 1.31 & 0.565 & 0.000 & 0.752 & 1.430 & (20) \\
\hline MME5 & TFSI & 2.44 & 25 & 6.540 & 14.31 & -4.33 & 200.1 & 179.3 & 1.30 & 0.585 & 0.000 & 0.995 & 1.544 & (20) \\
\hline MME6 & TFSI & 2.19 & 25 & 9.035 & 13.83 & -4.33 & 218.8 & 196.6 & 1.34 & 0.584 & 0.000 & 0.641 & 1.064 & (20) \\
\hline MMM5 & TFSI & 2.36 & 25 & 8.147 & 14.34 & -4.42 & 185.3 & 162.4 & 1.29 & 0.606 & 0.000 & 1.121 & 1.524 & (20) \\
\hline PRL15(c-C4N) & TFSI & 2.66 & 25 & 5.709 & 14.28 & -4.20 & 204.7 & 185.7 & 1.30 & 0.569 & 0.000 & 0.865 & 1.660 & (20) \\
\hline
\end{tabular}

\section{EOM: $\mathrm{CH} 2 \mathrm{CH} 2 \mathrm{OCH} 3 \quad$ TFSI: (CF3SO2)2N}

iP: isopropyl m-Me: meta-methyl

p-Me: para-methyl
$R_{2}-N_{1}^{R^{+}} \cdot$ Carbon\#
R 1 R 2R 3X
$\square^{+} \cdot$ Carbon\#
P ZX
$\mathrm{R}_{2} \mathrm{~N}^{\mathrm{N}=\mathrm{N}^{+} \mathrm{R}_{1} \quad \mathrm{R} 1 \mathrm{R} 2 \mathrm{I}}$
T ${ }^{+}$Carbon\#
PRLXX
$ح_{\text {Carbon\# }}^{\text {Carbon\# }}$
P PR X X
$R_{2}{ }^{-N} Y_{R_{3}}{ }^{N^{+}} \cdot R_{1}$
R 3 R 1 R 2 I 
Supporting Information. Table S2. Cation and Anion, Presence of Literature, and Experimental and Calculated Viscosities by Eq. (3).

\begin{tabular}{|c|c|c|c|c|c|c|c|c|c|c|c|c|c|c|}
\hline name & anion & logVis & $\mathrm{T}^{\circ} \mathrm{C}$ & $\mathrm{DP}$ & IP & LUMO & Area & Volume & Ovality & $\mathrm{N}$ & N2 & Eq.(3a) & $\mathrm{MR}(3 \mathrm{~b})$ & Lit. \\
\hline MEMI & TFSI & 1.944 & 20 & 1.536 & 14.53 & -4.88 & 248.7 & 165.7 & 1.70 & 0.404 & 0.385 & 1.547 & 1.597 & (15) \\
\hline BMI & BF4 & 2.367 & 30 & 5.935 & 14.60 & -4.95 & 252.1 & 182.0 & 1.62 & 0.415 & 0.403 & 1.999 & 1.956 & (15) \\
\hline MMI & TFSI & 1.643 & 20 & 0.915 & 15.03 & -5.06 & 177.3 & 113.3 & 1.57 & 0.416 & 0.416 & 1.405 & 1.474 & (15) \\
\hline EMI & BF4 & 1.576 & 25 & 1.757 & 14.95 & -4.98 & 217.6 & 143.7 & 1.64 & 0.416 & 0.394 & 1.800 & 1.814 & (15) \\
\hline BMI & PF6 & 2.494 & 30 & 5.935 & 14.60 & -4.95 & 252.1 & 182.0 & 1.62 & 0.415 & 0.403 & 2.311 & 2.348 & (15) \\
\hline EMI & CF3COO & 1.544 & 20 & 1.757 & 14.95 & -4.98 & 217.6 & 143.7 & 1.64 & 0.416 & 0.394 & 1.661 & 1.687 & (15) \\
\hline BMI & CF3COO & 1.863 & 20 & 5.935 & 14.60 & -4.95 & 252.1 & 182.0 & 1.62 & 0.415 & 0.403 & 1.943 & 1.931 & (15) \\
\hline BMI & TFSI & 1.716 & 20 & 5.935 & 14.60 & -4.95 & 252.1 & 182.0 & 1.62 & 0.415 & 0.403 & 1.792 & 1.803 & (15) \\
\hline BEI & C4F9SO3 & 2.509 & 20 & 4.421 & 14.57 & -4.86 & 235.3 & 178.2 & 1.54 & 0.408 & 0.399 & 2.559 & 2.587 & (15) \\
\hline EEI & CF3COO & 1.633 & 20 & 0.990 & 14.82 & -4.89 & 226.0 & 162.1 & 1.57 & 0.399 & 0.399 & 1.680 & 1.853 & (15) \\
\hline $\mathrm{BMI}$ & CF3SO3 & 1.954 & 20 & 5.935 & 14.60 & -4.95 & 252.1 & 182.0 & 1.62 & 0.415 & 0.403 & 1.997 & 2.047 & (15) \\
\hline BEI & CF3COO & 1.949 & 20 & 4.421 & 14.57 & -4.86 & 235.3 & 178.2 & 1.54 & 0.408 & 0.399 & 1.912 & 1.985 & (15) \\
\hline BMI & C4F9SO3 & 2.572 & 20 & 5.935 & 14.60 & -4.95 & 252.1 & 182.0 & 1.62 & 0.415 & 0.403 & 2.603 & 2.533 & (15) \\
\hline BMI & C3F7COO & 2.260 & 20 & 5.935 & 14.60 & -4.95 & 252.1 & 182.0 & 1.62 & 0.415 & 0.403 & 2.287 & 2.262 & (15) \\
\hline EMI & CF3SO3 & 1.630 & 25 & 1.757 & 14.95 & -4.98 & 217.6 & 143.7 & 1.64 & 0.416 & 0.394 & 1.617 & 1.700 & (15) \\
\hline EEI & TFSI & 1.544 & 20 & 0.990 & 14.82 & -4.89 & 226.0 & 162.1 & 1.57 & 0.399 & 0.399 & 1.554 & 1.725 & (15) \\
\hline EMI & TFSI & 1.531 & 20 & 1.757 & 14.95 & -4.98 & 217.6 & 143.7 & 1.64 & 0.416 & 0.394 & 1.537 & 1.559 & (15) \\
\hline EEI & CF3SO3 & 1.724 & 20 & 0.990 & 14.82 & -4.89 & 226.0 & 162.1 & 1.57 & 0.399 & 0.399 & 1.724 & 1.969 & (15) \\
\hline $8 \mathrm{MI}$ & PF6 & 2.673 & 25 & 14.952 & 13.20 & -4.92 & 310.6 & 262.0 & 1.57 & 0.414 & 0.413 & 2.892 & 2.921 & (17) \\
\hline BMI & TFSI & 1.233 & 45 & 5.935 & 14.60 & -4.95 & 252.1 & 182.0 & 1.62 & 0.415 & 0.403 & 1.390 & 1.290 & (17) \\
\hline BMI & TFSI & 1.464 & 30 & 5.935 & 14.60 & -4.95 & 252.1 & 182.0 & 1.62 & 0.415 & 0.403 & 1.612 & 1.598 & (17) \\
\hline BMI & TFSI & 1.325 & 40 & 5.935 & 14.60 & -4.95 & 252.1 & 182.0 & 1.62 & 0.415 & 0.403 & 1.458 & 1.393 & (17) \\
\hline BMI & TFSI & 1.399 & 35 & 5.935 & 14.60 & -4.95 & 252.1 & 182.0 & 1.62 & 0.415 & 0.403 & 1.532 & 1.495 & (17) \\
\hline BMI & TFSI & 1.207 & 50 & 5.935 & 14.60 & -4.95 & 252.1 & 182.0 & 1.62 & 0.415 & 0.403 & 1.326 & 1.187 & (17) \\
\hline PMI & TFSI & 1.547 & 25 & 3.777 & 14.92 & -4.97 & 239.8 & 177.4 & 1.57 & 0.415 & 0.403 & 1.659 & 1.720 & (17) \\
\hline $8 \mathrm{MI}$ & TFSI & 1.871 & 25 & 14.952 & 13.20 & -4.92 & 310.6 & 262.0 & 1.57 & 0.414 & 0.413 & 1.983 & 2.171 & (17) \\
\hline $10 \mathrm{MI}$ & TFSI & 1.957 & 25 & 19.997 & 12.79 & -4.92 & 357.4 & 290.0 & 1.69 & 0.414 & 0.412 & 2.032 & 2.244 & (17) \\
\hline EMI & TFSI & 1.398 & 25 & 1.757 & 14.95 & -4.98 & 217.6 & 143.7 & 1.64 & 0.416 & 0.394 & 1.460 & 1.456 & (17) \\
\hline $9 \mathrm{MI}$ & PF6 & 2.897 & 25 & 19.010 & 12.85 & -4.95 & 350.6 & 267.2 & 1.75 & 0.415 & 0.407 & 2.842 & 2.837 & (17) \\
\hline BMI & TFSI & 1.644 & 25 & 5.935 & 14.60 & -4.95 & 252.1 & 182.0 & 1.62 & 0.415 & 0.403 & 1.698 & 1.701 & (17) \\
\hline BMI & TFSI & 1.645 & 25 & 5.935 & 14.60 & -4.95 & 252.1 & 182.0 & 1.62 & 0.415 & 0.403 & 1.698 & 1.701 & (17) \\
\hline BMI & PF6 & 1.926 & 45 & 5.935 & 14.60 & -4.95 & 252.1 & 182.0 & 1.62 & 0.415 & 0.403 & 1.963 & 2.040 & (17) \\
\hline $6 \mathrm{MI}$ & TFSI & 1.772 & 25 & 11.084 & 13.62 & -4.95 & 301.4 & 220.1 & 1.71 & 0.414 & 0.407 & 1.802 & 1.832 & (17) \\
\hline $5 \mathrm{MI}$ & TFSI & 1.699 & 25 & 8.455 & 14.05 & -4.95 & 256.7 & 187.1 & 1.62 & 0.415 & 0.407 & 1.727 & 1.738 & (17) \\
\hline $7 \mathrm{MI}$ & PF6 & 2.636 & 25 & 13.616 & 13.30 & -4.95 & 321.2 & 233.3 & 1.75 & 0.415 & 0.407 & 2.661 & 2.625 & (17) \\
\hline BMI & PF6 & 2.094 & 40 & 5.935 & 14.60 & -4.95 & 252.1 & 182.0 & 1.62 & 0.415 & 0.403 & 2.070 & 2.142 & (17) \\
\hline BMI & PF6 & 1.844 & 50 & 5.935 & 14.60 & -4.95 & 252.1 & 182.0 & 1.62 & 0.415 & 0.403 & 1.864 & 1.937 & (17) \\
\hline $9 \mathrm{MI}$ & TFSI & 1.932 & 25 & 19.010 & 12.85 & -4.95 & 350.6 & 267.2 & 1.75 & 0.415 & 0.407 & 1.951 & 2.088 & (17) \\
\hline BMI & PF6 & 2.431 & 25 & 5.935 & 14.60 & -4.95 & 252.1 & 182.0 & 1.62 & 0.415 & 0.403 & 2.446 & 2.450 & (17) \\
\hline BMI & PF6 & 2.324 & 30 & 5.935 & 14.60 & -4.95 & 252.1 & 182.0 & 1.62 & 0.415 & 0.403 & 2.311 & 2.348 & (17) \\
\hline BMI & PF6 & 2.434 & 25 & 5.935 & 14.60 & -4.95 & 252.1 & 182.0 & 1.62 & 0.415 & 0.403 & 2.446 & 2.450 & (17) \\
\hline
\end{tabular}




\begin{tabular}{|c|c|c|c|c|c|c|c|c|c|c|c|c|c|c|}
\hline name & anion & logVis & $\mathrm{T}^{\circ} \mathrm{C}$ & $\mathrm{DP}$ & IP & LUMO & Area & Volume & Ovality & $\mathrm{N}$ & N2 & Eq.(3a) & $\mathrm{MR}(3 b)$ & Lit. \\
\hline BMI & PF6 & 2.196 & 35 & 5.935 & 14.60 & -4.95 & 252.1 & 182.0 & 1.62 & 0.415 & 0.403 & 2.186 & 2.245 & $(17)$ \\
\hline $6 \mathrm{MI}$ & PF6 & 2.611 & 25 & 11.084 & 13.62 & -4.95 & 301.4 & 220.1 & 1.71 & 0.414 & 0.407 & 2.609 & 2.581 & (17) \\
\hline 7MI & TFSI & 1.834 & 25 & 13.616 & 13.30 & -4.95 & 321.2 & 233.3 & 1.75 & 0.415 & 0.407 & 1.835 & 1.875 & (17) \\
\hline $5 \mathrm{MI}$ & PF6 & 2.491 & 25 & 8.455 & 14.05 & -4.95 & 256.7 & 187.1 & 1.62 & 0.415 & 0.407 & 2.492 & 2.487 & (17) \\
\hline PRL11(c-C4N) & CF3SO2NCOCF3 & 1.903 & 25 & 2.260 & 15.85 & -4.36 & 133.7 & 116.3 & 1.16 & 0.583 & 0.000 & 1.706 & 1.778 & (18) \\
\hline MMM3 & CF3SO2NCOCF3 & 1.653 & 25 & 3.449 & 15.76 & -4.45 & 147.6 & 127.8 & 1.20 & 0.606 & 0.000 & 1.781 & 1.767 & (18) \\
\hline MMMi3 & CF3SO2NCOCF3 & 1.954 & 25 & 2.040 & 16.81 & -4.40 & 139.6 & 124.9 & 1.16 & 0.584 & 0.000 & 1.835 & 1.846 & (18) \\
\hline EMI & TFSI & 1.531 & 25 & 1.757 & 14.95 & -4.98 & 217.6 & 143.7 & 1.64 & 0.416 & 0.394 & 1.460 & 1.456 & (18) \\
\hline EMI & CF3SO2NCOCF3 & 1.398 & 25 & 1.757 & 14.95 & -4.98 & 217.6 & 143.7 & 1.64 & 0.416 & 0.394 & 1.413 & 1.401 & (18) \\
\hline MMM3 & TFSI & 1.857 & 25 & 3.449 & 15.76 & -4.45 & 147.6 & 127.8 & 1.20 & 0.606 & 0.000 & 1.845 & 1.822 & (18) \\
\hline MMM2 & CF3SO2NCOCF3 & 1.708 & 25 & 1.408 & 16.87 & -4.49 & 127.1 & 108.0 & 1.16 & 0.599 & 0.000 & 1.713 & 1.760 & (18) \\
\hline MMM-All & CF3SO2NCOCF3 & 1.623 & 25 & 2.614 & 14.64 & -4.42 & 188.4 & 142.7 & 1.43 & 0.601 & 0.000 & 1.622 & 1.537 & (18) \\
\hline EEE2 & CF3SO2NCOCF3 & 1.778 & 25 & 1.010 & 15.91 & -4.22 & 166.4 & 155.6 & 1.19 & 0.540 & 0.000 & 1.778 & 1.924 & (18) \\
\hline MPMI & TFSI & 1.778 & 25 & 3.309 & 14.52 & -4.87 & 209.0 & 167.4 & 1.42 & 0.400 & 0.393 & 1.636 & 1.825 & (19) \\
\hline BMI & CF3COO & 1.863 & 20 & 5.935 & 14.60 & -4.95 & 252.1 & 182.0 & 1.62 & 0.415 & 0.403 & 1.943 & 1.931 & (19) \\
\hline BMI & TFSI & 1.716 & 20 & 5.935 & 14.60 & -4.95 & 252.1 & 182.0 & 1.62 & 0.415 & 0.403 & 1.792 & 1.803 & (19) \\
\hline BMI & CF3SO3 & 1.954 & 20 & 5.935 & 14.60 & -4.95 & 252.1 & 182.0 & 1.62 & 0.415 & 0.403 & 1.997 & 2.047 & (19) \\
\hline BMI & C4F9SO3 & 2.572 & 20 & 5.935 & 14.60 & -4.95 & 252.1 & 182.0 & 1.62 & 0.415 & 0.403 & 2.603 & 2.533 & (19) \\
\hline BMI & C3F7COO & 2.260 & 20 & 5.935 & 14.60 & -4.95 & 252.1 & 182.0 & 1.62 & 0.415 & 0.403 & 2.287 & 2.262 & (19) \\
\hline MPMI & TFSI & 1.778 & 25 & 3.309 & 14.52 & -4.87 & 209.0 & 167.4 & 1.42 & 0.400 & 0.393 & 1.636 & 1.825 & (19) \\
\hline BMI & CF3SO3 & 1.954 & 20 & 5.935 & 14.60 & -4.95 & 252.1 & 182.0 & 1.62 & 0.415 & 0.403 & 1.997 & 2.047 & (19) \\
\hline EMI & C3F7BF3 & 1.505 & 25 & 1.757 & 14.95 & -4.98 & 217.6 & 143.7 & 1.64 & 0.416 & 0.394 & 1.505 & 1.505 & (19) \\
\hline EMI & C4F9BF3 & 1.580 & 25 & 1.757 & 14.95 & -4.98 & 217.6 & 143.7 & 1.64 & 0.416 & 0.394 & 1.580 & 1.580 & (19) \\
\hline AllAIIN12 & TFSI & 2.044 & 25 & 0.639 & 14.38 & -4.22 & 222.4 & 191.4 & 1.38 & 0.563 & 0.000 & 1.698 & 1.847 & (20) \\
\hline MM66 & TFSI & 1.820 & 25 & 6.941 & 13.81 & -4.25 & 293.8 & 266.2 & 1.47 & 0.592 & 0.000 & 2.129 & 2.101 & (20) \\
\hline MM56 & TFSI & 1.816 & 25 & 6.744 & 13.82 & -4.25 & 275.0 & 248.9 & 1.44 & 0.592 & 0.000 & 2.092 & 2.055 & (20) \\
\hline MME9 & TFSI & 1.969 & 25 & 17.037 & 12.96 & -4.32 & 275.6 & 248.7 & 1.44 & 0.585 & 0.000 & 2.221 & 2.155 & (20) \\
\hline MME6 & TFSI & 1.813 & 25 & 9.035 & 13.83 & -4.33 & 218.8 & 196.6 & 1.34 & 0.584 & 0.000 & 2.043 & 1.978 & (20) \\
\hline PP66 & TFSI & 2.044 & 25 & 6.460 & 13.76 & -4.02 & 354.3 & 338.6 & 1.51 & 0.565 & 0.000 & 2.273 & 2.353 & (20) \\
\hline PRL17(c-C4N) & TFSI & 1.883 & 25 & 10.634 & 13.45 & -4.20 & 242.5 & 220.5 & 1.37 & 0.569 & 0.000 & 2.109 & 2.049 & (20) \\
\hline EEE7 & TFSI & 1.965 & 25 & 9.910 & 13.44 & -4.14 & 263.7 & 248.9 & 1.38 & 0.545 & 0.000 & 2.179 & 2.172 & (20) \\
\hline PRL44(c-C4N) & TFSI & 1.857 & 25 & 4.140 & 14.73 & -4.08 & 235.7 & 221.0 & 1.33 & 0.553 & 0.000 & 2.068 & 2.058 & (20) \\
\hline MME7 & TFSI & 1.895 & 25 & 11.649 & 13.47 & -4.32 & 237.9 & 214.0 & 1.37 & 0.585 & 0.000 & 2.105 & 2.031 & (20) \\
\hline PPB6 & TFSI & 1.972 & 25 & 5.161 & 13.77 & -4.03 & 316.8 & 304.0 & 1.45 & 0.565 & 0.000 & 2.175 & 2.250 & (20) \\
\hline MBB5 & TFSI & 1.874 & 25 & 3.799 & 14.23 & -4.15 & 286.8 & 266.8 & 1.43 & 0.580 & 0.000 & 2.074 & 2.113 & (20) \\
\hline MEE7 & TFSI & 1.934 & 25 & 10.477 & 13.45 & -4.24 & 251.4 & 230.9 & 1.38 & 0.565 & 0.000 & 2.133 & 2.093 & (20) \\
\hline MMP6 & TFSI & 1.843 & 25 & 7.128 & 13.82 & -4.29 & 237.6 & 213.9 & 1.37 & 0.589 & 0.000 & 2.031 & 1.983 & (20) \\
\hline MBB6 & TFSI & 1.846 & 25 & 3.314 & 13.79 & -4.15 & 306.2 & 283.9 & 1.47 & 0.580 & 0.000 & 2.031 & 2.135 & (20) \\
\hline MMB7 & TFSI & 1.894 & 25 & 7.493 & 13.45 & -4.27 & 275.3 & 248.5 & 1.44 & 0.588 & 0.000 & 2.076 & 2.056 & (20) \\
\hline MMB6 & TFSI & 1.813 & 25 & 4.939 & 13.81 & -4.27 & 256.5 & 231.2 & 1.41 & 0.588 & 0.000 & 1.994 & 1.997 & (20) \\
\hline MMP7 & TFSI & 1.922 & 25 & 9.708 & 13.46 & -4.28 & 256.6 & 231.3 & 1.41 & 0.589 & 0.000 & 2.100 & 2.040 & (20) \\
\hline PRL14(c-C4N) & TFSI & 1.736 & 25 & 3.455 & 14.89 & -4.21 & 185.8 & 168.3 & 1.26 & 0.569 & 0.000 & 1.907 & 1.917 & (20) \\
\hline
\end{tabular}




\begin{tabular}{|c|c|c|c|c|c|c|c|c|c|c|c|c|c|c|}
\hline name & anion & $\log V$ is & $\mathrm{T}^{\circ} \mathrm{C}$ & $\mathrm{DP}$ & IP & LUMO & Area & Volume & Ovality & $\mathrm{N}$ & $\mathrm{N} 2$ & Eq.(3a) & $M R(3 b)$ & Lit. \\
\hline PRL15(c-C4N) & TFSI & 1.808 & 25 & 5.709 & 14.28 & -4.20 & 204.7 & 185.7 & 1.30 & 0.569 & 0.000 & 1.980 & 1.952 & (20) \\
\hline MMM7 & TFSI & 1.927 & 25 & 13.348 & 13.49 & -4.42 & 223.2 & 197.2 & 1.36 & 0.607 & 0.000 & 2.083 & 1.978 & (20) \\
\hline MME5 & TFSI & 1.831 & 25 & 6.540 & 14.31 & -4.33 & 200.1 & 179.3 & 1.30 & 0.585 & 0.000 & 1.980 & 1.932 & (20) \\
\hline PRL24(c-C4N) & TFSI & 1.830 & 25 & 3.678 & 14.82 & -4.13 & 198.5 & 185.8 & 1.26 & 0.545 & 0.000 & 1.977 & 2.004 & (20) \\
\hline EEE5 & TFSI & 1.915 & 25 & 5.364 & 14.26 & -4.15 & 225.9 & 214.2 & 1.30 & 0.545 & 0.000 & 2.060 & 2.077 & (20) \\
\hline PRL34(c-C4N) & TFSI & 1.869 & 25 & 3.434 & 14.80 & -4.09 & 216.9 & 203.6 & 1.30 & 0.554 & 0.000 & 2.005 & 2.021 & (20) \\
\hline MME4 & TFSI & 2.041 & 25 & 4.128 & 14.92 & -4.34 & 181.1 & 161.9 & 1.26 & 0.585 & 0.000 & 1.910 & 1.895 & (20) \\
\hline 53EE & TFSI & 1.957 & 25 & 4.964 & 14.25 & -4.11 & 243.5 & 232.6 & 1.33 & 0.553 & 0.000 & 2.086 & 2.105 & (20) \\
\hline MMP3 & TFSI & 1.911 & 25 & 0.880 & 15.64 & -4.31 & 181.0 & 161.9 & 1.26 & 0.589 & 0.000 & 1.787 & 1.872 & (20) \\
\hline MEE5 & TFSI & 1.895 & 25 & 5.581 & 14.29 & -4.24 & 213.5 & 196.1 & 1.31 & 0.565 & 0.000 & 2.005 & 1.994 & (20) \\
\hline MEP5 & TFSI & 1.919 & 25 & 4.955 & 14.28 & -4.21 & 232.1 & 213.5 & 1.34 & 0.572 & 0.000 & 2.020 & 2.009 & (20) \\
\hline MMM5 & TFSI & 1.866 & 25 & 8.147 & 14.34 & -4.42 & 185.3 & 162.4 & 1.29 & 0.606 & 0.000 & 1.966 & 1.882 & (20) \\
\hline MPP5 & TFSI & 1.970 & 25 & 4.849 & 14.25 & -4.18 & 249.7 & 231.9 & 1.37 & 0.581 & 0.000 & 2.057 & 2.043 & (20) \\
\hline MMP5 & TFSI & 1.872 & 25 & 4.702 & 14.29 & -4.29 & 218.9 & 196.6 & 1.34 & 0.589 & 0.000 & 1.958 & 1.935 & (20) \\
\hline MEP3 & TFSI & 1.995 & 25 & 1.995 & 15.42 & -4.23 & 194.4 & 178.8 & 1.27 & 0.572 & 0.000 & 1.912 & 1.949 & (20) \\
\hline EEE3 & TFSI & 1.998 & 25 & 1.482 & 15.55 & -4.17 & 188.1 & 179.5 & 1.22 & 0.545 & 0.000 & 1.916 & 2.021 & (20) \\
\hline PZB & TFSI & 1.843 & 25 & 4.742 & 14.77 & -5.80 & 278.2 & 190.0 & 1.74 & 0.493 & 0.000 & 1.773 & 1.811 & (20) \\
\hline MMB5 & TFSI & 1.838 & 25 & 2.600 & 14.28 & -4.27 & 237.7 & 213.9 & 1.37 & 0.588 & 0.000 & 1.895 & 1.946 & (20) \\
\hline MME3 & TFSI & 1.878 & 25 & 1.952 & 15.70 & -4.35 & 162.3 & 144.6 & 1.22 & 0.585 & 0.000 & 1.829 & 1.871 & (20) \\
\hline MMP4 & TFSI & 1.898 & 25 & 2.351 & 14.90 & -4.30 & 199.8 & 179.1 & 1.30 & 0.589 & 0.000 & 1.864 & 1.895 & (20) \\
\hline MEE3 & TFSI & 1.922 & 25 & 1.855 & 15.63 & -4.26 & 174.9 & 162.2 & 1.22 & 0.565 & 0.000 & 1.888 & 1.953 & (20) \\
\hline PRL33(c-C4N) & TFSI & 1.957 & 25 & 2.008 & 15.27 & -4.12 & 198.1 & 186.2 & 1.26 & 0.557 & 0.000 & 1.933 & 1.990 & (20) \\
\hline PPR12(c-C5N) & TFSI & 2.336 & 25 & 2.474 & 15.74 & -4.22 & 160.5 & 151.1 & 1.17 & 0.566 & 0.000 & 1.920 & 1.963 & (22) \\
\hline PPR14(c-C5N) & TFSI & 2.330 & 25 & 3.700 & 14.86 & -4.17 & 195.8 & 183.5 & 1.25 & 0.576 & 0.000 & 1.974 & 1.972 & (22) \\
\hline EMI & BF4 & 1.505 & 25 & 1.757 & 14.95 & -4.98 & 217.6 & 143.7 & 1.64 & 0.416 & 0.394 & 1.800 & 1.814 & (22) \\
\hline MEE-EOM & BF4 & 2.629 & 25 & 4.531 & 14.23 & -4.31 & 182.5 & 165.5 & 1.25 & 0.569 & 0.000 & 2.352 & 2.279 & (22) \\
\hline MME-EOM & BF4 & 2.525 & 25 & 4.613 & 14.29 & -4.40 & 169.4 & 146.8 & 1.26 & 0.593 & 0.000 & 2.262 & 2.180 & (22) \\
\hline MMI & TFSI & 1.580 & 25 & 0.915 & 15.03 & -5.06 & 177.3 & 113.3 & 1.57 & 0.416 & 0.416 & 1.336 & 1.371 & (22) \\
\hline MEE4 & CF3BF3 & 2.322 & 25 & 3.766 & 14.87 & -4.22 & 191.4 & 172.1 & 1.28 & 0.565 & 0.000 & 2.082 & 2.025 & (22) \\
\hline MMI & C2F5BF3 & 1.519 & 25 & 0.915 & 15.03 & -5.06 & 177.3 & 113.3 & 1.57 & 0.416 & 0.416 & 1.306 & 1.325 & (22) \\
\hline $\mathrm{MI}-\mathrm{CH} 2 \mathrm{OM}$ & C2F5BF3 & 1.672 & 25 & 3.152 & 14.82 & -4.94 & 202.8 & 137.6 & 1.57 & 0.414 & 0.372 & 1.482 & 1.473 & (22) \\
\hline EMI & $\mathrm{CH} 3 \mathrm{COO}$ & 2.210 & 20 & 1.757 & 14.95 & -4.98 & 217.6 & 143.7 & 1.64 & 0.416 & 0.394 & 2.390 & 2.445 & (22) \\
\hline PMI & CF3BF3 & 1.633 & 25 & 3.777 & 14.92 & -4.97 & 239.8 & 177.4 & 1.57 & 0.415 & 0.403 & 1.789 & 1.824 & (22) \\
\hline EMI & CF3BF3 & 1.415 & 25 & 1.757 & 14.95 & -4.98 & 217.6 & 143.7 & 1.64 & 0.416 & 0.394 & 1.570 & 1.561 & (22) \\
\hline MEE4 & TFSI & 2.079 & 25 & 3.766 & 14.87 & -4.22 & 191.4 & 172.1 & 1.28 & 0.565 & 0.000 & 1.925 & 1.921 & (22) \\
\hline PRL1-CH2OM(c-C4N) & C2F5BF3 & 1.568 & 25 & 1.787 & 15.30 & -4.27 & 161.9 & 140.9 & 1.24 & 0.545 & 0.000 & 1.718 & 1.813 & (22) \\
\hline BMI & BF4 & 2.255 & 25 & 5.935 & 14.60 & -4.95 & 252.1 & 182.0 & 1.62 & 0.415 & 0.403 & 2.112 & 2.059 & (22) \\
\hline BMI & CF3BF3 & 1.690 & 25 & 5.935 & 14.60 & -4.95 & 252.1 & 182.0 & 1.62 & 0.415 & 0.403 & 1.833 & 1.805 & (22) \\
\hline MEE4 & C2F5BF3 & 2.017 & 25 & 3.766 & 14.87 & -4.22 & 191.4 & 172.1 & 1.28 & 0.565 & 0.000 & 1.876 & 1.875 & (22) \\
\hline PPR13(c-C5N) & TFSI & 2.068 & 25 & 2.649 & 15.56 & -4.18 & 176.7 & 165.9 & 1.21 & 0.573 & 0.000 & 1.950 & 1.961 & (22) \\
\hline EMI & CF3COO & 1.544 & 20 & 1.757 & 14.95 & -4.98 & 217.6 & 143.7 & 1.64 & 0.416 & 0.394 & 1.661 & 1.687 & (22) \\
\hline MI-EOM & CF3BF3 & 1.633 & 25 & 6.392 & 13.91 & -5.01 & 246.2 & 171.3 & 1.65 & 0.415 & 0.408 & 1.743 & 1.714 & (22) \\
\hline
\end{tabular}




\begin{tabular}{|c|c|c|c|c|c|c|c|c|c|c|c|c|c|c|}
\hline name & anion & logVis & $\mathrm{T}^{\circ} \mathrm{C}$ & $\mathrm{DP}$ & IP & LUMO & Area & Volume & Ovality & $\mathrm{N}$ & $\mathrm{N} 2$ & Eq.(3a) & $M R(3 b)$ & Lit. \\
\hline EEE-EOM & CF3BF3 & 2.179 & 25 & 4.120 & 14.14 & -4.23 & 195.2 & 181.7 & 1.26 & 0.553 & 0.000 & 2.070 & 2.086 & (22) \\
\hline $\mathrm{MI}-\mathrm{CH} 2 \mathrm{OM}$ & CF3BF3 & 1.740 & 25 & 3.152 & 14.82 & -4.94 & 202.8 & 137.6 & 1.57 & 0.414 & 0.372 & 1.634 & 1.624 & (22) \\
\hline MMM4 & TFSI & 1.991 & 25 & 5.689 & 14.96 & -4.43 & 164.2 & 142.1 & 1.25 & 0.606 & 0.000 & 1.894 & 1.833 & (22) \\
\hline PZB & BF4 & 2.114 & 25 & 4.742 & 14.77 & -5.80 & 278.2 & 190.0 & 1.74 & 0.493 & 0.000 & 2.211 & 2.169 & (22) \\
\hline MEE3 & TFSI & 1.973 & 25 & 1.855 & 15.63 & -4.26 & 174.9 & 162.2 & 1.22 & 0.565 & 0.000 & 1.888 & 1.953 & (22) \\
\hline EMI & C3F7COO & 2.021 & 20 & 1.757 & 14.95 & -4.98 & 217.6 & 143.7 & 1.64 & 0.416 & 0.394 & 1.943 & 2.017 & (22) \\
\hline EEE-EOM & C2F5BF3 & 1.940 & 25 & 4.120 & 14.14 & -4.23 & 195.2 & 181.7 & 1.26 & 0.553 & 0.000 & 1.865 & 1.936 & (22) \\
\hline PMI & C2F5BF3 & 1.544 & 25 & 3.777 & 14.92 & -4.97 & 239.8 & 177.4 & 1.57 & 0.415 & 0.403 & 1.618 & 1.673 & (22) \\
\hline EMI & TFSI & 1.519 & 25 & 1.757 & 14.95 & -4.98 & 217.6 & 143.7 & 1.64 & 0.416 & 0.394 & 1.460 & 1.456 & (22) \\
\hline PMI & BF4 & 2.013 & 25 & 3.777 & 14.92 & -4.97 & 239.8 & 177.4 & 1.57 & 0.415 & 0.403 & 2.061 & 2.078 & (22) \\
\hline BMI & C2F5BF3 & 1.613 & 25 & 5.935 & 14.60 & -4.95 & 252.1 & 182.0 & 1.62 & 0.415 & 0.403 & 1.656 & 1.655 & (22) \\
\hline PRL1-EOM(c-C4N) & C2F5BF3 & 1.716 & 25 & 3.489 & 14.24 & -4.28 & 177.6 & 156.8 & 1.26 & 0.576 & 0.000 & 1.759 & 1.804 & (22) \\
\hline MEE-EOM & TFSI & 1.839 & 25 & 4.531 & 14.23 & -4.31 & 182.5 & 165.5 & 1.25 & 0.569 & 0.000 & 1.880 & 1.921 & (22) \\
\hline $\mathrm{MMM}-\mathrm{CH} 2 \mathrm{OM}$ & TFSI & 1.699 & 25 & 3.116 & 15.39 & -4.50 & 141.1 & 116.5 & 1.22 & 0.582 & 0.000 & 1.734 & 1.771 & (22) \\
\hline MME-EOM & TFSI & 1.778 & 25 & 4.613 & 14.29 & -4.40 & 169.4 & 146.8 & 1.26 & 0.593 & 0.000 & 1.812 & 1.822 & (22) \\
\hline MME-EOM & CF3BF3 & 1.987 & 25 & 4.613 & 14.29 & -4.40 & 169.4 & 146.8 & 1.26 & 0.593 & 0.000 & 1.958 & 1.926 & (22) \\
\hline PRL13(c-C4N) & TFSI & 1.799 & 25 & 1.524 & 15.67 & -4.23 & 167.0 & 151.0 & 1.22 & 0.569 & 0.000 & 1.828 & 1.896 & (22) \\
\hline EMI & CF3SO3 & 1.633 & 25 & 1.757 & 14.95 & -4.98 & 217.6 & 143.7 & 1.64 & 0.416 & 0.394 & 1.617 & 1.700 & (22) \\
\hline EEE-EOM & TFSI & 1.929 & 25 & 4.120 & 14.14 & -4.23 & 195.2 & 181.7 & 1.26 & 0.553 & 0.000 & 1.914 & 1.982 & (22) \\
\hline EMI & CF3SO2NCOCF3 & 1.398 & 25 & 1.757 & 14.95 & -4.98 & 217.6 & 143.7 & 1.64 & 0.416 & 0.394 & 1.413 & 1.401 & (22) \\
\hline MMM3 & TFSI & 1.857 & 25 & 3.449 & 15.76 & -4.45 & 147.6 & 127.8 & 1.20 & 0.606 & 0.000 & 1.845 & 1.822 & (22) \\
\hline PRL14(c-C4N) & C2F5BF3 & 1.851 & 25 & 3.455 & 14.89 & -4.21 & 185.8 & 168.3 & 1.26 & 0.569 & 0.000 & 1.858 & 1.871 & (22) \\
\hline EMI & C2F5BF3 & 1.431 & 25 & 1.757 & 14.95 & -4.98 & 217.6 & 143.7 & 1.64 & 0.416 & 0.394 & 1.425 & 1.410 & (22) \\
\hline PZB & TFSI & 1.778 & 25 & 4.742 & 14.77 & -5.80 & 278.2 & 190.0 & 1.74 & 0.493 & 0.000 & 1.773 & 1.811 & (22) \\
\hline MMI & CF3BF3 & 1.431 & 25 & 0.915 & 15.03 & -5.06 & 177.3 & 113.3 & 1.57 & 0.416 & 0.416 & 1.434 & 1.476 & (22) \\
\hline MME-EOM & C2F5BF3 & 1.763 & 25 & 4.613 & 14.29 & -4.40 & 169.4 & 146.8 & 1.26 & 0.593 & 0.000 & 1.766 & 1.775 & (22) \\
\hline MI-EOM & C2F5BF3 & 1.580 & 25 & 6.392 & 13.91 & -5.01 & 246.2 & 171.3 & 1.65 & 0.415 & 0.408 & 1.578 & 1.564 & (22) \\
\hline MEE-EOM & CF3BF3 & 2.033 & 25 & 4.531 & 14.23 & -4.31 & 182.5 & 165.5 & 1.25 & 0.569 & 0.000 & 2.033 & 2.025 & (22) \\
\hline EMI & C3F7BF3 & 1.505 & 25 & 1.757 & 14.95 & -4.98 & 217.6 & 143.7 & 1.64 & 0.416 & 0.394 & 1.505 & 1.505 & (22) \\
\hline EMI & C4F9BF3 & 1.580 & 25 & 1.757 & 14.95 & -4.98 & 217.6 & 143.7 & 1.64 & 0.416 & 0.394 & 1.580 & 1.580 & (22) \\
\hline MEE-EOM & C2F5BF3 & 1.833 & 25 & 4.531 & 14.23 & -4.31 & 182.5 & 165.5 & 1.25 & 0.569 & 0.000 & 1.833 & 1.875 & (22) \\
\hline AllAllI & $\mathrm{Cl}$ & 3.078 & 25 & 1.788 & 14.13 & -4.75 & 337.8 & 226.5 & 1.88 & 0.400 & 0.400 & 3.255 & 3.385 & (22) \\
\hline AllEI & $\mathrm{Br}$ & 2.859 & 25 & 1.860 & 14.17 & -4.83 & 235.5 & 178.3 & 1.54 & 0.401 & 0.393 & 2.990 & 3.059 & (22) \\
\hline AlIMI & $\mathrm{Br}$ & 2.930 & 25 & 2.932 & 14.19 & -4.90 & 270.4 & 172.6 & 1.80 & 0.414 & 0.401 & 2.900 & 2.752 & (22) \\
\hline AllAllI & TFSI & 1.491 & 27 & 1.788 & 14.13 & -4.75 & 337.8 & 226.5 & 1.88 & 0.400 & 0.400 & 1.515 & 1.576 & (22) \\
\hline AllAllI & $\mathrm{Br}$ & 2.918 & 27 & 1.788 & 14.13 & -4.75 & 337.8 & 226.5 & 1.88 & 0.400 & 0.400 & 2.936 & 2.912 & (22) \\
\hline AllPI & $\mathrm{Br}$ & 3.221 & 25 & 2.043 & 14.16 & -4.81 & 311.2 & 238.3 & 1.67 & 0.402 & 0.401 & 3.221 & 3.206 & (22) \\
\hline BBB8 & CF3SO3 & 3.307 & 25 & 7.376 & 13.14 & -4.02 & 386.9 & 362.8 & 1.57 & 0.564 & 0.000 & 2.530 & 2.646 & (24) \\
\hline BBB6 & TFSI & 2.775 & 25 & 3.736 & 13.76 & -4.02 & 349.7 & 328.6 & 1.52 & 0.564 & 0.000 & 2.130 & 2.280 & (24) \\
\hline BBB7 & TFSI & 2.782 & 25 & 5.465 & 13.41 & -4.02 & 368.3 & 345.7 & 1.55 & 0.564 & 0.000 & 2.195 & 2.338 & (24) \\
\hline BBB8 & TFSI & 2.759 & 25 & 7.376 & 13.14 & -4.02 & 386.9 & 362.8 & 1.57 & 0.564 & 0.000 & 2.258 & 2.402 & (24) \\
\hline HMI & $\mathrm{Cl}$ & 4.257 & 25 & 10.144 & 13.79 & -4.92 & 294.4 & 219.8 & 1.67 & 0.414 & 0.412 & 3.902 & 3.622 & (24) \\
\hline
\end{tabular}




\begin{tabular}{|c|c|c|c|c|c|c|c|c|c|c|c|c|c|c|}
\hline name & anion & logVis & $\mathrm{T}^{\circ} \mathrm{C}$ & $\mathrm{DP}$ & IP & LUMO & Area & Volume & Ovality & $\mathrm{N}$ & N2 & Eq.(3a) & $M R(3 b)$ & Lit. \\
\hline EiPiP7 & TFSI & 2.559 & 25 & 8.984 & 13.40 & -4.04 & 279.2 & 274.0 & 1.37 & 0.531 & 0.000 & 2.231 & 2.267 & (24) \\
\hline MPMI & TFSI & 1.954 & 25 & 3.309 & 14.52 & -4.87 & 209.0 & 167.4 & 1.42 & 0.400 & 0.393 & 1.636 & 1.825 & (24) \\
\hline EEE7 & TFSI & 1.878 & 25 & 9.910 & 13.44 & -4.14 & 263.7 & 248.9 & 1.38 & 0.545 & 0.000 & 2.179 & 2.172 & (24) \\
\hline MHMI & TFSI & 2.501 & 10 & 10.002 & 13.62 & -4.85 & 271.0 & 221.9 & 1.53 & 0.400 & 0.394 & 2.204 & 2.315 & (24) \\
\hline PZ2 & EtOSO3 & 1.021 & 30 & 0.660 & 15.47 & -5.84 & 244.4 & 153.0 & 1.77 & 0.484 & 0.000 & 1.296 & 1.105 & (24) \\
\hline HMI & $\mathrm{Cl}$ & 3.535 & 40 & 10.144 & 13.79 & -4.92 & 294.4 & 219.8 & 1.67 & 0.414 & 0.412 & 3.262 & 3.314 & (24) \\
\hline MHMI & TFSI & 2.233 & 20 & 10.002 & 13.62 & -4.85 & 271.0 & 221.9 & 1.53 & 0.400 & 0.394 & 1.965 & 2.110 & (24) \\
\hline MHMI & TFSI & 2.117 & 25 & 10.002 & 13.62 & -4.85 & 271.0 & 221.9 & 1.53 & 0.400 & 0.394 & 1.860 & 2.007 & (24) \\
\hline EMI & BF4 & 1.656 & 20 & 1.757 & 14.95 & -4.98 & 217.6 & 143.7 & 1.64 & 0.416 & 0.394 & 1.901 & 1.917 & (24) \\
\hline MHMI & TFSI & 2.004 & 30 & 10.002 & 13.62 & -4.85 & 271.0 & 221.9 & 1.53 & 0.400 & 0.394 & 1.763 & 1.904 & (24) \\
\hline EMI & BF4 & 1.570 & 25 & 1.757 & 14.95 & -4.98 & 217.6 & 143.7 & 1.64 & 0.416 & 0.394 & 1.800 & 1.814 & (24) \\
\hline PZ2 & EtOSO3 & 1.137 & 25 & 0.660 & 15.47 & -5.84 & 244.4 & 153.0 & 1.77 & 0.484 & 0.000 & 1.360 & 1.208 & (24) \\
\hline HMI & $\mathrm{Cl}$ & 2.974 & 55 & 10.144 & 13.79 & -4.92 & 294.4 & 219.8 & 1.67 & 0.414 & 0.412 & 2.755 & 3.006 & (24) \\
\hline EMI & BF4 & 1.408 & 35 & 1.757 & 14.95 & -4.98 & 217.6 & 143.7 & 1.64 & 0.416 & 0.394 & 1.620 & 1.609 & (24) \\
\hline EMI & BF4 & 1.496 & 30 & 1.757 & 14.95 & -4.98 & 217.6 & 143.7 & 1.64 & 0.416 & 0.394 & 1.707 & 1.712 & (24) \\
\hline MHMI & TFSI & 1.799 & 40 & 10.002 & 13.62 & -4.85 & 271.0 & 221.9 & 1.53 & 0.400 & 0.394 & 1.590 & 1.699 & (24) \\
\hline EMI & BF4 & 1.333 & 40 & 1.757 & 14.95 & -4.98 & 217.6 & 143.7 & 1.64 & 0.416 & 0.394 & 1.541 & 1.506 & (24) \\
\hline EMI & BF4 & 1.201 & 50 & 1.757 & 14.95 & -4.98 & 217.6 & 143.7 & 1.64 & 0.416 & 0.394 & 1.400 & 1.301 & (24) \\
\hline EMI & BF4 & 1.275 & 45 & 1.757 & 14.95 & -4.98 & 217.6 & 143.7 & 1.64 & 0.416 & 0.394 & 1.468 & 1.404 & (24) \\
\hline MHMI & TFSI & 1.623 & 50 & 10.002 & 13.62 & -4.85 & 271.0 & 221.9 & 1.53 & 0.400 & 0.394 & 1.443 & 1.494 & (24) \\
\hline HMI & $\mathrm{Cl}$ & 2.531 & 70 & 10.144 & 13.79 & -4.92 & 294.4 & 219.8 & 1.67 & 0.414 & 0.412 & 2.352 & 2.698 & (24) \\
\hline BMI & BF4 & 1.060 & 80 & 5.935 & 14.60 & -4.95 & 252.1 & 182.0 & 1.62 & 0.415 & 0.403 & 1.238 & 0.929 & (24) \\
\hline MMM6 & TFSI & 2.185 & 25 & 10.692 & 13.85 & -4.42 & 201.4 & 176.4 & 1.32 & 0.607 & 0.000 & 2.013 & 1.913 & (24) \\
\hline PZ2 & EtOSO3 & 1.262 & 20 & 0.660 & 15.47 & -5.84 & 244.4 & 153.0 & 1.77 & 0.484 & 0.000 & 1.430 & 1.311 & (24) \\
\hline MHMI & TFSI & 1.477 & 60 & 10.002 & 13.62 & -4.85 & 271.0 & 221.9 & 1.53 & 0.400 & 0.394 & 1.316 & 1.288 & (24) \\
\hline BMI & BF4 & 1.193 & 70 & 5.935 & 14.60 & -4.95 & 252.1 & 182.0 & 1.62 & 0.415 & 0.403 & 1.349 & 1.135 & (24) \\
\hline $8 \mathrm{MI}$ & PF6 & 3.807 & 0 & 14.952 & 13.20 & -4.92 & 310.6 & 262.0 & 1.57 & 0.414 & 0.413 & 3.956 & 3.434 & (24) \\
\hline HMI & PF6 & 2.767 & 25 & 10.144 & 13.79 & -4.92 & 294.4 & 219.8 & 1.67 & 0.414 & 0.412 & 2.622 & 2.604 & (24) \\
\hline BMI & BF4 & 1.333 & 60 & 5.935 & 14.60 & -4.95 & 252.1 & 182.0 & 1.62 & 0.415 & 0.403 & 1.476 & 1.340 & (24) \\
\hline BMI & TFSI & 1.839 & 25 & 5.935 & 14.60 & -4.95 & 252.1 & 182.0 & 1.62 & 0.415 & 0.403 & 1.698 & 1.701 & (24) \\
\hline $\mathrm{PZZ}(\mathrm{m}-\mathrm{Me})$ & TFSI & 1.431 & 50 & 13.697 & 13.10 & -5.65 & 389.3 & 282.3 & 1.87 & 0.511 & 0.000 & 1.567 & 1.663 & (24) \\
\hline MHMI & TFSI & 1.342 & 70 & 10.002 & 13.62 & -4.85 & 271.0 & 221.9 & 1.53 & 0.400 & 0.394 & 1.207 & 1.083 & (24) \\
\hline BMI & BF4 & 1.492 & 50 & 5.935 & 14.60 & -4.95 & 252.1 & 182.0 & 1.62 & 0.415 & 0.403 & 1.624 & 1.545 & (24) \\
\hline BMI & BF4 & 1.577 & 45 & 5.935 & 14.60 & -4.95 & 252.1 & 182.0 & 1.62 & 0.415 & 0.403 & 1.707 & 1.648 & (24) \\
\hline BMI & BF4 & 1.668 & 40 & 5.935 & 14.60 & -4.95 & 252.1 & 182.0 & 1.62 & 0.415 & 0.403 & 1.797 & 1.751 & (24) \\
\hline BMI & BF4 & 1.765 & 35 & 5.935 & 14.60 & -4.95 & 252.1 & 182.0 & 1.62 & 0.415 & 0.403 & 1.894 & 1.853 & (24) \\
\hline BMI & BF4 & 1.870 & 30 & 5.935 & 14.60 & -4.95 & 252.1 & 182.0 & 1.62 & 0.415 & 0.403 & 1.999 & 1.956 & (24) \\
\hline MMM8 & TFSI & 2.258 & 25 & 16.047 & 13.20 & -4.42 & 238.6 & 210.6 & 1.39 & 0.607 & 0.000 & 2.130 & 2.024 & (24) \\
\hline $8 \mathrm{MI}$ & PF6 & 3.590 & 5 & 14.952 & 13.20 & -4.92 & 310.6 & 262.0 & 1.57 & 0.414 & 0.413 & 3.706 & 3.331 & (24) \\
\hline EEE6 & TFSI & 2.223 & 25 & 8.264 & 13.79 & -4.16 & 239.5 & 225.2 & 1.34 & 0.543 & 0.000 & 2.120 & 2.111 & (24) \\
\hline MMM7 & TFSI & 2.185 & 25 & 13.348 & 13.49 & -4.42 & 223.2 & 197.2 & 1.36 & 0.607 & 0.000 & 2.083 & 1.978 & (24) \\
\hline $8 \mathrm{MI}$ & PF6 & 3.387 & 10 & 14.952 & 13.20 & -4.92 & 310.6 & 262.0 & 1.57 & 0.414 & 0.413 & 3.476 & 3.229 & (24) \\
\hline
\end{tabular}




\begin{tabular}{|c|c|c|c|c|c|c|c|c|c|c|c|c|c|c|}
\hline name & anion & $\log V$ is & $\mathrm{T}^{\circ} \mathrm{C}$ & $\mathrm{DP}$ & IP & LUMO & Area & Volume & Ovality & $\mathrm{N}$ & $\mathrm{N} 2$ & Eq.(3a) & $M R(3 b)$ & Lit. \\
\hline BMI & PF6 & 3.235 & 0 & 5.935 & 14.60 & -4.95 & 252.1 & 182.0 & 1.62 & 0.415 & 0.403 & 3.322 & 2.963 & (24) \\
\hline EEE8 & TFSI & 2.305 & 25 & 12.926 & 13.16 & -4.16 & 278.2 & 260.3 & 1.41 & 0.547 & 0.000 & 2.229 & 2.213 & (24) \\
\hline BMI & PF6 & 3.049 & 5 & 5.935 & 14.60 & -4.95 & 252.1 & 182.0 & 1.62 & 0.415 & 0.403 & 3.116 & 2.861 & (24) \\
\hline $\mathrm{PZH}$ & TFSI & 2.276 & 10 & 9.715 & 13.73 & -5.79 & 346.5 & 229.1 & 1.91 & 0.494 & 0.000 & 2.212 & 2.185 & (24) \\
\hline $\mathrm{PZB}(\mathrm{m}-\mathrm{Me})$ & BF4 & 1.362 & 70 & 3.769 & 14.73 & -5.66 & 319.0 & 221.1 & 1.80 & 0.511 & 0.000 & 1.423 & 1.297 & (24) \\
\hline $8 \mathrm{MI}$ & BF4 & 2.159 & 40 & 14.952 & 13.20 & -4.92 & 310.6 & 262.0 & 1.57 & 0.414 & 0.413 & 2.103 & 2.221 & (24) \\
\hline 8MI & BF4 & 1.948 & 50 & 14.952 & 13.20 & -4.92 & 310.6 & 262.0 & 1.57 & 0.414 & 0.413 & 1.893 & 2.016 & (24) \\
\hline $8 \mathrm{MI}$ & BF4 & 2.275 & 35 & 14.952 & 13.20 & -4.92 & 310.6 & 262.0 & 1.57 & 0.414 & 0.413 & 2.221 & 2.324 & (24) \\
\hline $\mathrm{PZH}$ & TFSI & 2.025 & 20 & 9.715 & 13.73 & -5.79 & 346.5 & 229.1 & 1.91 & 0.494 & 0.000 & 1.972 & 1.979 & (24) \\
\hline $8 \mathrm{MI}$ & BF4 & 2.400 & 30 & 14.952 & 13.20 & -4.92 & 310.6 & 262.0 & 1.57 & 0.414 & 0.413 & 2.349 & 2.426 & (24) \\
\hline BMI & PF6 & 2.878 & 10 & 5.935 & 14.60 & -4.95 & 252.1 & 182.0 & 1.62 & 0.415 & 0.403 & 2.927 & 2.758 & (24) \\
\hline 8MI & BF4 & 1.762 & 60 & 14.952 & 13.20 & -4.92 & 310.6 & 262.0 & 1.57 & 0.414 & 0.413 & 1.713 & 1.811 & (24) \\
\hline PMI & BF4 & 2.013 & 25 & 3.777 & 14.92 & -4.97 & 239.8 & 177.4 & 1.57 & 0.415 & 0.403 & 2.061 & 2.078 & (24) \\
\hline $8 \mathrm{MI}$ & BF4 & 2.533 & 25 & 14.952 & 13.20 & -4.92 & 310.6 & 262.0 & 1.57 & 0.414 & 0.413 & 2.487 & 2.529 & (24) \\
\hline EMI & TFSI & 1.505 & 25 & 1.757 & 14.95 & -4.98 & 217.6 & 143.7 & 1.64 & 0.416 & 0.394 & 1.460 & 1.456 & (24) \\
\hline $\mathrm{PZH}(\mathrm{m}-\mathrm{Me})$ & TFSI & 1.204 & 70 & 8.535 & 13.70 & -5.66 & 364.2 & 258.7 & 1.86 & 0.511 & 0.000 & 1.248 & 1.108 & (24) \\
\hline $8 \mathrm{MI}$ & PF6 & 3.027 & 20 & 14.952 & 13.20 & -4.92 & 310.6 & 262.0 & 1.57 & 0.414 & 0.413 & 3.071 & 3.023 & (24) \\
\hline $\mathrm{PZB}(\mathrm{m}-\mathrm{Me})$ & BF4 & 1.519 & 60 & 3.769 & 14.73 & -5.66 & 319.0 & 221.1 & 1.80 & 0.511 & 0.000 & 1.560 & 1.502 & (24) \\
\hline $\mathrm{PZB}(\mathrm{m}-\mathrm{Me})$ & BF4 & 1.681 & 50 & 3.769 & 14.73 & -5.66 & 319.0 & 221.1 & 1.80 & 0.511 & 0.000 & 1.720 & 1.708 & (24) \\
\hline BMI & $\mathrm{CH} 3 \mathrm{COO}$ & 1.987 & 50 & 5.935 & 14.60 & -4.95 & 252.1 & 182.0 & 1.62 & 0.415 & 0.403 & 2.025 & 2.074 & (24) \\
\hline 8MI & BF4 & 2.675 & 20 & 14.952 & 13.20 & -4.92 & 310.6 & 262.0 & 1.57 & 0.414 & 0.413 & 2.637 & 2.632 & (24) \\
\hline BMI & $\mathrm{CH} 3 \mathrm{COO}$ & 1.623 & 70 & 5.935 & 14.60 & -4.95 & 252.1 & 182.0 & 1.62 & 0.415 & 0.403 & 1.661 & 1.663 & (24) \\
\hline $\mathrm{PZH}$ & TFSI & 1.806 & 30 & 9.715 & 13.73 & -5.79 & 346.5 & 229.1 & 1.91 & 0.494 & 0.000 & 1.769 & 1.774 & (24) \\
\hline BMI & $\mathrm{CH} 3 \mathrm{COO}$ & 1.792 & 60 & 5.935 & 14.60 & -4.95 & 252.1 & 182.0 & 1.62 & 0.415 & 0.403 & 1.830 & 1.868 & (24) \\
\hline $8 \mathrm{MI}$ & BF4 & 1.595 & 70 & 14.952 & 13.20 & -4.92 & 310.6 & 262.0 & 1.57 & 0.414 & 0.413 & 1.558 & 1.605 & (24) \\
\hline PZ2 & EtOSO3 & 1.551 & 10 & 0.660 & 15.47 & -5.84 & 244.4 & 153.0 & 1.77 & 0.484 & 0.000 & 1.589 & 1.516 & (24) \\
\hline $\mathrm{PZH}$ & TFSI & 1.903 & 25 & 9.715 & 13.73 & -5.79 & 346.5 & 229.1 & 1.91 & 0.494 & 0.000 & 1.866 & 1.877 & (24) \\
\hline BMI & PF6 & 2.717 & 15 & 5.935 & 14.60 & -4.95 & 252.1 & 182.0 & 1.62 & 0.415 & 0.403 & 2.753 & 2.656 & (24) \\
\hline BMI & $\mathrm{CH} 3 \mathrm{COO}$ & 2.217 & 40 & 5.935 & 14.60 & -4.95 & 252.1 & 182.0 & 1.62 & 0.415 & 0.403 & 2.253 & 2.279 & (24) \\
\hline $\mathrm{PZB}(\mathrm{m}-\mathrm{Me})$ & BF4 & 2.713 & 10 & 3.769 & 14.73 & -5.66 & 319.0 & 221.1 & 1.80 & 0.511 & 0.000 & 2.679 & 2.529 & (24) \\
\hline BMI & $\mathrm{CH} 3 \mathrm{COO}$ & 2.490 & 30 & 5.935 & 14.60 & -4.95 & 252.1 & 182.0 & 1.62 & 0.415 & 0.403 & 2.520 & 2.484 & (24) \\
\hline EMI & TFSI & 0.954 & 70 & 1.757 & 14.95 & -4.98 & 217.6 & 143.7 & 1.64 & 0.416 & 0.394 & 0.983 & 0.532 & (24) \\
\hline $8 \mathrm{MI}$ & BF4 & 2.828 & 15 & 14.952 & 13.20 & -4.92 & 310.6 & 262.0 & 1.57 & 0.414 & 0.413 & 2.799 & 2.734 & (24) \\
\hline $\mathrm{PZH}$ & TFSI & 1.623 & 40 & 9.715 & 13.73 & -5.79 & 346.5 & 229.1 & 1.91 & 0.494 & 0.000 & 1.595 & 1.569 & (24) \\
\hline $\mathrm{PZB}(\mathrm{m}-\mathrm{Me})$ & TFSI & 1.146 & 70 & 3.769 & 14.73 & -5.66 & 319.0 & 221.1 & 1.80 & 0.511 & 0.000 & 1.173 & 0.939 & (24) \\
\hline HMI & TFSI & 2.170 & 10 & 10.144 & 13.79 & -4.92 & 294.4 & 219.8 & 1.67 & 0.414 & 0.412 & 2.143 & 2.163 & (24) \\
\hline 8MI & BF4 & 1.516 & 75 & 14.952 & 13.20 & -4.92 & 310.6 & 262.0 & 1.57 & 0.414 & 0.413 & 1.489 & 1.503 & (24) \\
\hline 8MI & PF6 & 2.866 & 25 & 14.952 & 13.20 & -4.92 & 310.6 & 262.0 & 1.57 & 0.414 & 0.413 & 2.892 & 2.921 & (24) \\
\hline BMI & $\mathrm{CH} 3 \mathrm{COO}$ & 2.643 & 25 & 5.935 & 14.60 & -4.95 & 252.1 & 182.0 & 1.62 & 0.415 & 0.403 & 2.670 & 2.587 & (24) \\
\hline BMI & PF6 & 2.567 & 20 & 5.935 & 14.60 & -4.95 & 252.1 & 182.0 & 1.62 & 0.415 & 0.403 & 2.593 & 2.553 & (24) \\
\hline EMI & TFSI & 1.415 & 30 & 1.757 & 14.95 & -4.98 & 217.6 & 143.7 & 1.64 & 0.416 & 0.394 & 1.389 & 1.354 & (24) \\
\hline $\mathrm{PZZ}(\mathrm{m}-\mathrm{Me})$ & TFSI & 1.279 & 70 & 13.697 & 13.10 & -5.65 & 389.3 & 282.3 & 1.87 & 0.511 & 0.000 & 1.304 & 1.252 & (24) \\
\hline
\end{tabular}




\begin{tabular}{|c|c|c|c|c|c|c|c|c|c|c|c|c|c|c|}
\hline name & anion & logVis & $\mathrm{T}^{\circ} \mathrm{C}$ & $\mathrm{DP}$ & IP & LUMO & Area & Volume & Ovality & $\mathrm{N}$ & N2 & Eq.(3a) & $\mathrm{MR}(3 \mathrm{~b})$ & Lit. \\
\hline $8 \mathrm{MI}$ & BF4 & 1.449 & 80 & 14.952 & 13.20 & -4.92 & 310.6 & 262.0 & 1.57 & 0.414 & 0.413 & 1.424 & 1.400 & (24) \\
\hline BMI & CF3COO & 1.279 & 60 & 5.935 & 14.60 & -4.95 & 252.1 & 182.0 & 1.62 & 0.415 & 0.403 & 1.303 & 1.110 & (24) \\
\hline HMI & TFSI & 1.740 & 30 & 10.144 & 13.79 & -4.92 & 294.4 & 219.8 & 1.67 & 0.414 & 0.412 & 1.716 & 1.752 & (24) \\
\hline $\mathrm{PZB}(\mathrm{m}-\mathrm{Me})$ & TFSI & 1.255 & 60 & 3.769 & 14.73 & -5.66 & 319.0 & 221.1 & 1.80 & 0.511 & 0.000 & 1.278 & 1.144 & (24) \\
\hline BMI & $\mathrm{CH} 3 \mathrm{COO}$ & 2.810 & 20 & 5.935 & 14.60 & -4.95 & 252.1 & 182.0 & 1.62 & 0.415 & 0.403 & 2.833 & 2.690 & (24) \\
\hline HMI & TFSI & 1.934 & 20 & 10.144 & 13.79 & -4.92 & 294.4 & 219.8 & 1.67 & 0.414 & 0.412 & 1.912 & 1.957 & (24) \\
\hline HMI & TFSI & 1.833 & 25 & 10.144 & 13.79 & -4.92 & 294.4 & 219.8 & 1.67 & 0.414 & 0.412 & 1.810 & 1.855 & (24) \\
\hline EMI & TFSI & 1.176 & 50 & 1.757 & 14.95 & -4.98 & 217.6 & 143.7 & 1.64 & 0.416 & 0.394 & 1.155 & 0.943 & (24) \\
\hline $\mathrm{PZH}(\mathrm{m}-\mathrm{Me})$ & TFSI & 1.342 & 60 & 8.535 & 13.70 & -5.66 & 364.2 & 258.7 & 1.86 & 0.511 & 0.000 & 1.363 & 1.313 & (24) \\
\hline $\mathrm{PZ8}(\mathrm{m}-\mathrm{Me})$ & TFSI & 1.944 & 30 & 13.697 & 13.10 & -5.65 & 389.3 & 282.3 & 1.87 & 0.511 & 0.000 & 1.924 & 2.073 & (24) \\
\hline $8 \mathrm{MI}$ & PF6 & 1.991 & 60 & 14.952 & 13.20 & -4.92 & 310.6 & 262.0 & 1.57 & 0.414 & 0.413 & 1.971 & 2.202 & (24) \\
\hline BMI & CF3COO & 1.176 & 70 & 5.935 & 14.60 & -4.95 & 252.1 & 182.0 & 1.62 & 0.415 & 0.403 & 1.196 & 0.905 & (24) \\
\hline EMI & TFSI & 1.556 & 20 & 1.757 & 14.95 & -4.98 & 217.6 & 143.7 & 1.64 & 0.416 & 0.394 & 1.537 & 1.559 & (24) \\
\hline 8MI & BF4 & 3.360 & 0 & 14.952 & 13.20 & -4.92 & 310.6 & 262.0 & 1.57 & 0.414 & 0.413 & 3.379 & 3.042 & (24) \\
\hline BMI & CF3COO & 1.724 & 30 & 5.935 & 14.60 & -4.95 & 252.1 & 182.0 & 1.62 & 0.415 & 0.403 & 1.743 & 1.726 & (24) \\
\hline $\mathrm{PZB}(\mathrm{m}-\mathrm{Me})$ & TFSI & 1.380 & 50 & 3.769 & 14.73 & -5.66 & 319.0 & 221.1 & 1.80 & 0.511 & 0.000 & 1.399 & 1.350 & (24) \\
\hline HMI & TFSI & 1.568 & 40 & 10.144 & 13.79 & -4.92 & 294.4 & 219.8 & 1.67 & 0.414 & 0.412 & 1.550 & 1.547 & (24) \\
\hline $8 \mathrm{MI}$ & PF6 & 1.804 & 70 & 14.952 & 13.20 & -4.92 & 310.6 & 262.0 & 1.57 & 0.414 & 0.413 & 1.786 & 1.997 & (24) \\
\hline $\mathrm{PZH}(\mathrm{m}-\mathrm{Me})$ & TFSI & 1.477 & 50 & 8.535 & 13.70 & -5.66 & 364.2 & 258.7 & 1.86 & 0.511 & 0.000 & 1.495 & 1.519 & (24) \\
\hline $8 \mathrm{MI}$ & PF6 & 2.202 & 50 & 14.952 & 13.20 & -4.92 & 310.6 & 262.0 & 1.57 & 0.414 & 0.413 & 2.185 & 2.407 & (24) \\
\hline $\mathrm{PZ8}(\mathrm{m}-\mathrm{Me})$ & TFSI & 1.748 & 40 & 13.697 & 13.10 & -5.65 & 389.3 & 282.3 & 1.87 & 0.511 & 0.000 & 1.732 & 1.868 & (24) \\
\hline $\mathrm{PZZ}(\mathrm{m}-\mathrm{Me})$ & TFSI & 2.049 & 25 & 13.697 & 13.10 & -5.65 & 389.3 & 282.3 & 1.87 & 0.511 & 0.000 & 2.033 & 2.176 & (24) \\
\hline EMI & TFSI & 1.079 & 60 & 1.757 & 14.95 & -4.98 & 217.6 & 143.7 & 1.64 & 0.416 & 0.394 & 1.063 & 0.738 & (24) \\
\hline EMI & TFSI & 1.279 & 40 & 1.757 & 14.95 & -4.98 & 217.6 & 143.7 & 1.64 & 0.416 & 0.394 & 1.263 & 1.148 & (24) \\
\hline $8 \mathrm{MI}$ & BF4 & 2.992 & 10 & 14.952 & 13.20 & -4.92 & 310.6 & 262.0 & 1.57 & 0.414 & 0.413 & 2.976 & 2.837 & (24) \\
\hline $\mathrm{PZH}$ & TFSI & 1.462 & 50 & 9.715 & 13.73 & -5.79 & 346.5 & 229.1 & 1.91 & 0.494 & 0.000 & 1.447 & 1.363 & (24) \\
\hline BMI & PF6 & 2.200 & 35 & 5.935 & 14.60 & -4.95 & 252.1 & 182.0 & 1.62 & 0.415 & 0.403 & 2.186 & 2.245 & (24) \\
\hline $\mathrm{PZZ}(\mathrm{m}-\mathrm{Me})$ & TFSI & 2.164 & 20 & 13.697 & 13.10 & -5.65 & 389.3 & 282.3 & 1.87 & 0.511 & 0.000 & 2.150 & 2.279 & (24) \\
\hline $\mathrm{PZB}(\mathrm{m}-\mathrm{Me})$ & BF4 & 1.892 & 40 & 3.769 & 14.73 & -5.66 & 319.0 & 221.1 & 1.80 & 0.511 & 0.000 & 1.906 & 1.913 & (24) \\
\hline BMI & CF3COO & 1.415 & 50 & 5.935 & 14.60 & -4.95 & 252.1 & 182.0 & 1.62 & 0.415 & 0.403 & 1.428 & 1.315 & (24) \\
\hline $\mathrm{PZB}(\mathrm{m}-\mathrm{Me})$ & BF4 & 2.391 & 20 & 3.769 & 14.73 & -5.66 & 319.0 & 221.1 & 1.80 & 0.511 & 0.000 & 2.378 & 2.324 & (24) \\
\hline 8MI & PF6 & 2.715 & 30 & 14.952 & 13.20 & -4.92 & 310.6 & 262.0 & 1.57 & 0.414 & 0.413 & 2.728 & 2.818 & (24) \\
\hline BMI & CF3COO & 2.190 & 10 & 5.935 & 14.60 & -4.95 & 252.1 & 182.0 & 1.62 & 0.415 & 0.403 & 2.179 & 2.137 & (24) \\
\hline $\mathrm{PZZ}(\mathrm{m}-\mathrm{Me})$ & TFSI & 2.428 & 10 & 13.697 & 13.10 & -5.65 & 389.3 & 282.3 & 1.87 & 0.511 & 0.000 & 2.417 & 2.484 & (24) \\
\hline $\mathrm{PZZ}(\mathrm{m}-\mathrm{Me})$ & TFSI & 1.415 & 60 & 13.697 & 13.10 & -5.65 & 389.3 & 282.3 & 1.87 & 0.511 & 0.000 & 1.426 & 1.457 & (24) \\
\hline $\mathrm{PZB}(\mathrm{m}-\mathrm{Me})$ & TFSI & 2.140 & 10 & 3.769 & 14.73 & -5.66 & 319.0 & 221.1 & 1.80 & 0.511 & 0.000 & 2.130 & 2.171 & (24) \\
\hline BMI & $\mathrm{CH} 3 \mathrm{COO}$ & 3.212 & 10 & 5.935 & 14.60 & -4.95 & 252.1 & 182.0 & 1.62 & 0.415 & 0.403 & 3.202 & 2.895 & (24) \\
\hline BMI & PF6 & 2.436 & 25 & 5.935 & 14.60 & -4.95 & 252.1 & 182.0 & 1.62 & 0.415 & 0.403 & 2.446 & 2.450 & (24) \\
\hline BMI & PF6 & 1.545 & 70 & 5.935 & 14.60 & -4.95 & 252.1 & 182.0 & 1.62 & 0.415 & 0.403 & 1.536 & 1.526 & (24) \\
\hline $\mathrm{PZB}(\mathrm{m}-\mathrm{Me})$ & TFSI & 1.531 & 40 & 3.769 & 14.73 & -5.66 & 319.0 & 221.1 & 1.80 & 0.511 & 0.000 & 1.541 & 1.555 & (24) \\
\hline BMI & PF6 & 2.079 & 40 & 5.935 & 14.60 & -4.95 & 252.1 & 182.0 & 1.62 & 0.415 & 0.403 & 2.070 & 2.142 & (24) \\
\hline HMI & TFSI & 1.415 & 50 & 10.144 & 13.79 & -4.92 & 294.4 & 219.8 & 1.67 & 0.414 & 0.412 & 1.407 & 1.341 & (24) \\
\hline
\end{tabular}




\begin{tabular}{|c|c|c|c|c|c|c|c|c|c|c|c|c|c|c|}
\hline name & anion & logVis & $\mathrm{T}^{\circ} \mathrm{C}$ & $\mathrm{DP}$ & IP & LUMO & Area & Volume & Ovality & $\mathrm{N}$ & $\mathrm{N} 2$ & Eq.(3a) & $M R(3 b)$ & Lit. \\
\hline $8 \mathrm{MI}$ & $\mathrm{Cl}$ & 4.320 & 25 & 14.952 & 13.20 & -4.92 & 310.6 & 262.0 & 1.57 & 0.414 & 0.413 & 4.327 & 3.939 & (24) \\
\hline 8MI & PF6 & 2.442 & 40 & 14.952 & 13.20 & -4.92 & 310.6 & 262.0 & 1.57 & 0.414 & 0.413 & 2.435 & 2.613 & (24) \\
\hline $\mathrm{PZH}(\mathrm{m}-\mathrm{Me})$ & TFSI & 1.643 & 40 & 8.535 & 13.70 & -5.66 & 364.2 & 258.7 & 1.86 & 0.511 & 0.000 & 1.650 & 1.724 & (24) \\
\hline $\mathrm{PZH}$ & TFSI & 1.204 & 70 & 9.715 & 13.73 & -5.79 & 346.5 & 229.1 & 1.91 & 0.494 & 0.000 & 1.211 & 0.953 & (24) \\
\hline BMI & CF3COO & 1.949 & 20 & 5.935 & 14.60 & -4.95 & 252.1 & 182.0 & 1.62 & 0.415 & 0.403 & 1.943 & 1.931 & (24) \\
\hline HMI & TFSI & 1.279 & 60 & 10.144 & 13.79 & -4.92 & 294.4 & 219.8 & 1.67 & 0.414 & 0.412 & 1.285 & 1.136 & (24) \\
\hline BMI & CF3COO & 1.845 & 25 & 5.935 & 14.60 & -4.95 & 252.1 & 182.0 & 1.62 & 0.415 & 0.403 & 1.839 & 1.829 & (24) \\
\hline BMI & PF6 & 2.305 & 30 & 5.935 & 14.60 & -4.95 & 252.1 & 182.0 & 1.62 & 0.415 & 0.403 & 2.311 & 2.348 & (24) \\
\hline $\mathrm{PZH}(\mathrm{m}-\mathrm{Me})$ & TFSI & 1.826 & 30 & 8.535 & 13.70 & -5.66 & 364.2 & 258.7 & 1.86 & 0.511 & 0.000 & 1.831 & 1.929 & (24) \\
\hline BMI & CF3COO & 1.568 & 40 & 5.935 & 14.60 & -4.95 & 252.1 & 182.0 & 1.62 & 0.415 & 0.403 & 1.573 & 1.521 & (24) \\
\hline EMI & TFSI & 1.716 & 10 & 1.757 & 14.95 & -4.98 & 217.6 & 143.7 & 1.64 & 0.416 & 0.394 & 1.712 & 1.764 & (24) \\
\hline 8MI & $\mathrm{Cl}$ & 3.038 & 55 & 14.952 & 13.20 & -4.92 & 310.6 & 262.0 & 1.57 & 0.414 & 0.413 & 3.042 & 3.323 & (24) \\
\hline $\mathrm{PZH}(\mathrm{m}-\mathrm{Me})$ & TFSI & 1.929 & 25 & 8.535 & 13.70 & -5.66 & 364.2 & 258.7 & 1.86 & 0.511 & 0.000 & 1.933 & 2.032 & (24) \\
\hline HMI & TFSI & 1.176 & 70 & 10.144 & 13.79 & -4.92 & 294.4 & 219.8 & 1.67 & 0.414 & 0.412 & 1.179 & 0.931 & (24) \\
\hline $\mathrm{PZB}(\mathrm{m}-\mathrm{Me})$ & TFSI & 1.903 & 20 & 3.769 & 14.73 & -5.66 & 319.0 & 221.1 & 1.80 & 0.511 & 0.000 & 1.900 & 1.966 & (24) \\
\hline $\mathrm{PZB}(\mathrm{m}-\mathrm{Me})$ & BF4 & 2.248 & 25 & 3.769 & 14.73 & -5.66 & 319.0 & 221.1 & 1.80 & 0.511 & 0.000 & 2.245 & 2.221 & (24) \\
\hline BMI & PF6 & 1.867 & 50 & 5.935 & 14.60 & -4.95 & 252.1 & 182.0 & 1.62 & 0.415 & 0.403 & 1.864 & 1.937 & (24) \\
\hline $\mathrm{PZB}(\mathrm{m}-\mathrm{Me})$ & BF4 & 2.121 & 30 & 3.769 & 14.73 & -5.66 & 319.0 & 221.1 & 1.80 & 0.511 & 0.000 & 2.123 & 2.118 & (24) \\
\hline BMI & PF6 & 1.690 & 60 & 5.935 & 14.60 & -4.95 & 252.1 & 182.0 & 1.62 & 0.415 & 0.403 & 1.688 & 1.732 & (24) \\
\hline $\mathrm{PZH}(\mathrm{m}-\mathrm{Me})$ & TFSI & 2.041 & 20 & 8.535 & 13.70 & -5.66 & 364.2 & 258.7 & 1.86 & 0.511 & 0.000 & 2.044 & 2.135 & (24) \\
\hline BMI & PF6 & 1.407 & 80 & 5.935 & 14.60 & -4.95 & 252.1 & 182.0 & 1.62 & 0.415 & 0.403 & 1.404 & 1.321 & (24) \\
\hline $8 \mathrm{MI}$ & PF6 & 2.574 & 35 & 14.952 & 13.20 & -4.92 & 310.6 & 262.0 & 1.57 & 0.414 & 0.413 & 2.576 & 2.715 & (24) \\
\hline $\mathrm{PZH}$ & TFSI & 1.322 & 60 & 9.715 & 13.73 & -5.79 & 346.5 & 229.1 & 1.91 & 0.494 & 0.000 & 1.320 & 1.158 & (24) \\
\hline $8 \mathrm{MI}$ & $\mathrm{Cl}$ & 2.588 & 70 & 14.952 & 13.20 & -4.92 & 310.6 & 262.0 & 1.57 & 0.414 & 0.413 & 2.590 & 3.015 & (24) \\
\hline $\mathrm{PZB}(\mathrm{m}-\mathrm{Me})$ & TFSI & 1.708 & 30 & 3.769 & 14.73 & -5.66 & 319.0 & 221.1 & 1.80 & 0.511 & 0.000 & 1.706 & 1.760 & (24) \\
\hline $8 \mathrm{MI}$ & BF4 & 3.169 & 5 & 14.952 & 13.20 & -4.92 & 310.6 & 262.0 & 1.57 & 0.414 & 0.413 & 3.169 & 2.940 & (24) \\
\hline $\mathrm{PZH}(\mathrm{m}-\mathrm{Me})$ & TFSI & 2.294 & 10 & 8.535 & 13.70 & -5.66 & 364.2 & 258.7 & 1.86 & 0.511 & 0.000 & 2.294 & 2.340 & (24) \\
\hline $\mathrm{PZ2}(\mathrm{m}-\mathrm{Me})$ & EtOSO3 & 1.617 & 10 & 1.114 & 14.88 & -5.70 & 296.5 & 179.6 & 1.93 & 0.502 & 0.000 & 1.617 & 1.450 & (24) \\
\hline $8 \mathrm{MI}$ & $\mathrm{Cl}$ & 3.609 & 40 & 14.952 & 13.20 & -4.92 & 310.6 & 262.0 & 1.57 & 0.414 & 0.413 & 3.609 & 3.631 & (24) \\
\hline $\mathrm{PZB}(\mathrm{m}-\mathrm{Me})$ & TFSI & 1.799 & 25 & 3.769 & 14.73 & -5.66 & 319.0 & 221.1 & 1.80 & 0.511 & 0.000 & 1.799 & 1.863 & (24) \\
\hline \multicolumn{15}{|l|}{ Prediction Data Set } \\
\hline AllEI & TFSI & 1.462 & 27 & 1.860 & 14.17 & -4.83 & 235.5 & 178.3 & 1.54 & 0.401 & 0.393 & 1.508 & 1.682 & $(22)$ \\
\hline EMI & $\mathrm{Cl}$ & 1.787 & 80 & 1.757 & 14.95 & -4.98 & 217.6 & 143.7 & 1.64 & 0.416 & 0.394 & 1.700 & 2.094 & (22) \\
\hline HMI & BF4 & 2.35 & 25 & 10.144 & 13.79 & -4.92 & 294.4 & 219.8 & 1.67 & 0.414 & 0.412 & 2.260 & 2.213 & (22) \\
\hline M888 & CF3COO & 3.365 & 20 & 10.441 & 13.14 & -4.13 & 486.6 & 451.3 & 1.71 & 0.580 & 0.000 & 2.823 & 2.941 & (22) \\
\hline M888 & TFSI & 2.802 & 20 & 10.441 & 13.14 & -4.13 & 486.6 & 451.3 & 1.71 & 0.580 & 0.000 & 2.587 & 2.813 & (22) \\
\hline MBMI & BF4 & 2.334 & 40 & 5.358 & 14.51 & -4.85 & 233.5 & 183.4 & 1.50 & 0.400 & 0.393 & 1.828 & 1.891 & (22) \\
\hline MHMI & BF4 & 3.053 & 20 & 10.002 & 13.62 & -4.85 & 271.0 & 221.9 & 1.53 & 0.400 & 0.394 & 2.463 & 2.468 & (22) \\
\hline PRL14(c-C4N) & CF3SO3 & 2.24 & 25 & 3.455 & 14.89 & -4.21 & 185.8 & 168.3 & 1.26 & 0.569 & 0.000 & 2.128 & 2.161 & (22) \\
\hline PZB & PF6 & 1.544 & 80 & 4.742 & 14.77 & -5.80 & 278.2 & 190.0 & 1.74 & 0.493 & 0.000 & 1.462 & 1.431 & (22) \\
\hline$P Z B(p-M e)$ & BF4 & 2.393 & 25 & 3.653 & 14.71 & -5.67 & 272.2 & 212.6 & 1.58 & 0.470 & 0.000 & 2.311 & 2.436 & (22) \\
\hline $\mathrm{PZH}$ & BF4 & 2.743 & 20 & 9.715 & 13.73 & -5.79 & 346.5 & 229.1 & 1.91 & 0.494 & 0.000 & 2.472 & 2.337 & (22) \\
\hline
\end{tabular}




\begin{tabular}{|c|c|c|c|c|c|c|c|c|c|c|c|c|c|}
\hline nam & anion & logVis & $\mathrm{T}^{\circ} \mathrm{C}$ & DP & IP & LUMO & Area & Volume & Ovality & $\mathrm{N}$ & N2 & Eq.(3a & $\mathrm{MR}(3 \mathrm{~b})$ \\
\hline $\mathrm{PZH}$ & PF6 & 2.347 & 50 & 9.715 & 13.73 & -5.79 & 346.5 & 229.1 & 1.91 & 0.494 & 0.000 & 2.054 & $2.113 \quad(22$ \\
\hline
\end{tabular}

m-Me: meta-methyl

p-Me: para-methyl 